\title{
The Role of Clt1-Regulated Xylan Metabolism in Melanin and Toxin Formation for the Pathogenicity of Curvularia lunata in Maize
}

\author{
Jinxin Gao, ${ }^{1,2}$ and Jie Chen ${ }^{1, \dagger}$ \\ ${ }^{1}$ School of Agriculture and Biology, State Key Laboratory of Microbial Metabolism, and Ministry of Agriculture Key Laboratory \\ of Urban Agriculture (South), Shanghai Jiao Tong University, 800 Dongchuan Road, Shanghai 200240, P. R. China \\ ${ }^{2}$ Department of Biology, New York University, New York, NY 10003, U.S.A.
}

Accepted 6 January 2021.

\begin{abstract}
We previously reported that the BTB (brica-brac, tramtrack, and broad) domain-containing protein Clt1 regulates melanin and toxin synthesis, conidiation, and pathogenicity in Curvularia lunata, but the interacting proteins and regulative mechanism of Clt1 are unclear. In this research, we identified two proteins, which respectively correspond to xylanase (Clxyn24) and acetyl xylan esterase (Claxe43) from $C$. lunata, that were regulated by Clt1. Yeast two-hybrid (Y2H) and bimolecular fluorescence complementation assays were conducted to verify the interaction of Clt1 with full-length Clxyn24 and Claxe43. Furthermore, the Y2H assay revealed that Clt1 physically interacted with Clxyn24 and Claxe43 through its BTB domain to degrade xylan, which was used as a carbon source for C. lunata growth. The utilization of xylan provides acetyl-CoA for the synthesis of melanin and toxin as well as energy and other intermediate metabolites for conidiation. Furthermore, transcriptome analysis revealed that $P K S 18$ and its 13 flanking genes found clustered in a region spanning $57.89 \mathrm{~kb}$ on scaffold 9 of the $C$. lunata $\mathrm{CX}-3$ genome were down-regulated in toxin production-deficient mutant $\mathbf{T} 806$, and this cluster is possibly responsible for toxin biosynthesis of $C$. lunata.
\end{abstract}

Keywords: Clt1, Curvularia lunata, melanin, toxin, xylan

Curvularia lunata (Wakker) Boedijn 1933 (teleomorph: Cochliobolus lunatus) is known as a human and plant pathogen (Gao et al. 2017; Khan et al. 2017). Curvularia leaf spot (CLS), caused by $C$. lunata, is one of the most widely distributed maize leaf diseases in the world (Gao et al. 2012, 2015a; Liu et al. 2016; Xu et al. 2007). In addition, C. lunata can also infect the

${ }^{\dagger}$ Corresponding author: J. Chen; jiechen59@sjtu.edu.cn

Funding: Financial support was provided by the National Natural Science Foundation of China (number 31872015), Key National R \& D Programs-Key International Intergovernmental Scientific and Technological Innovation Cooperation Projects (number 2017YFD0200403), and China Agriculture Research System (number CARS-02).

*The $e$-Xtra logo stands for "electronic extra" and indicates there is supplementary material published online.

The author(s) declare no conflict of interest. maize ear, particularly when a serious outbreak of the disease occurs in the field. Therefore, some contamination of the ear by M5HF2C toxin in infected grains poses a potential risk to the health of humans and livestock (Gao et al. 2017).

To uncover the pathogenicity mechanism of CLS, scientists have focused on identifying virulence factors, such as melanin and toxin (Gao et al. 2014b). The melanin enables the fungi to survive in the extreme environments, especially protecting them against host resistance during infection. Furthermore, melanin increases the penetration of appressoria and infection pegs that are necessary for host infection (Chumley and Valent 1990; Gao et al. 2015c). The 1,8-dihydroxynaphthalene (DHN) melanin is one of the major melanins produced by the plant-pathogenic fungi (Wang et al. 2019). DHN melanin biosynthesis starts from acetyl CoA or malonyl $\mathrm{CoA}$ and involves multiple enzymes, including the polyketide synthase (PKS), scytalone dehydratase (SCD1), 1,3,8-naphthalenetriol reductase (BRN1), and hydroxynaphthalene reductase (BRN2) (Gao and Chen 2017; Rižner and Wheeler 2003). Deletion of the NADPH oxidase gene causes partial reduction of DHN melanin by inhibiting the expression of melanin biosynthesis genes (Wang et al. 2020).

Previous work by our group has successfully identified methyl 5-(hydroxymethyl) furan-2-carboxylate (M5HF2C) toxin produced by C. lunata (Liu et al. 2009). Compared with melanin, the production mechanism of the toxin is still unknown. Although CLT1 has been reported to be involved in multiple cellular processes in $C$. lunata, especially as a key factor for melanin and toxin synthesis (Gao et al. 2014a), the regulation mode of CLT1 in M5HF2C toxin production is unknown.

To gain further insight into the melanin and toxin production mechanism, the interacting proteins of Clt1 were screened and characterized. The phenotypic analysis showed that interaction of Clt1 with Clxyn24 and Claxe43 is required for melanin and toxin formation, conidiation, and pathogenicity in $C$. lunata. This study provided new insights into the regulative mechanism of melanin and toxin synthesis of $C$. lunata and may lead to the discovery of the target site for controlling CLS.

\section{RESULTS}

Screening proteins regulated by Clt1.

Previously, we showed that a BTB (brica-brac, tramtrack, and broad) domain-containing protein Clt1 contributed to toxin production, the formation of melanin and conidiation, and pathogenicity of $C$. lunata. To understand the mechanism of Clt1mediated melanin and toxin formation and pathogenicity, we 
screened interacting proteins with $\mathrm{Clt} 1$ in a $C$. lunata cDNA library, using a yeast two-hybrid $(\mathrm{Y} 2 \mathrm{H})$ method. The yeast strain AH109, carrying an empty vector of a plasmid for CLT1 expression, was cultured on yeast peptone dextrose adenine media, to test self-activation and toxicity of Clt1 protein to yeast cells, before performing the $\mathrm{Y} 2 \mathrm{H}$ assay. The $\mathrm{Y} 2 \mathrm{H}$ screen was carried out on the cDNA library, and eight proteins were captured successfully (Supplementary Table S1). One of the Clt1-interacting proteins is xylanase which was designated ClXyn24 (GenBank accession number XM_007698555), and another interacting protein was identified as acetyl xylan esterase and was named ClAxe43 (GenBank accession number XM_007684984). Other proteins identified are listed in Supplementary Table S1.

The CLXYN24 and CLAXE43 genes were selected for characterization. Phylogenetic analysis showed that the Clxyn24 protein falls in a dothideomycete group of xylanase homologs (Supplementary Fig. S1A). Alignment of Clxyn24 with Setosphaeria turcica Stxyn showed a 94\% identity (Supplementary Fig. S1B). Alignment of xylanase homologs from C. lunata and S. turcica using ClustalW identified a conserved glycosyl hydrolase domain,

A
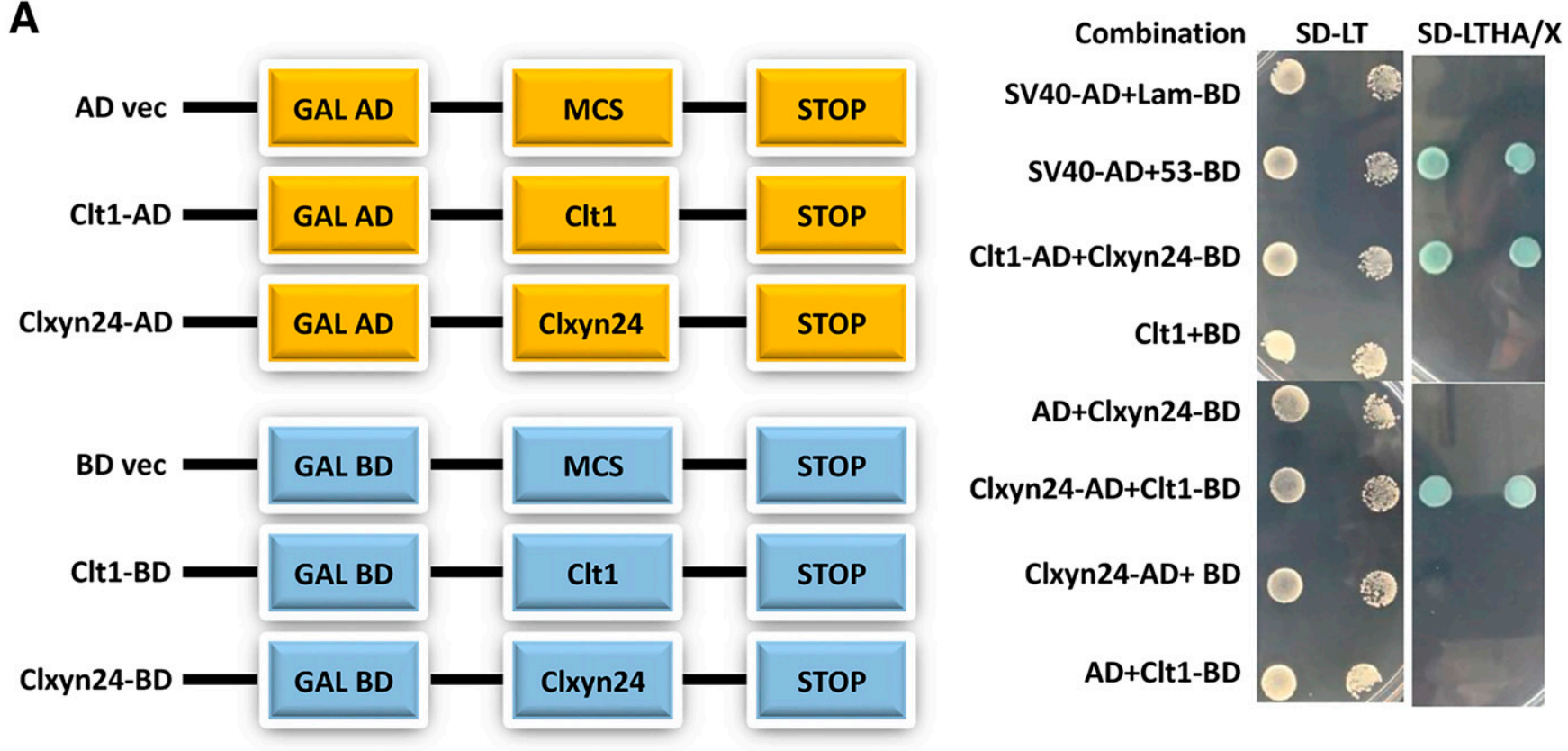

B

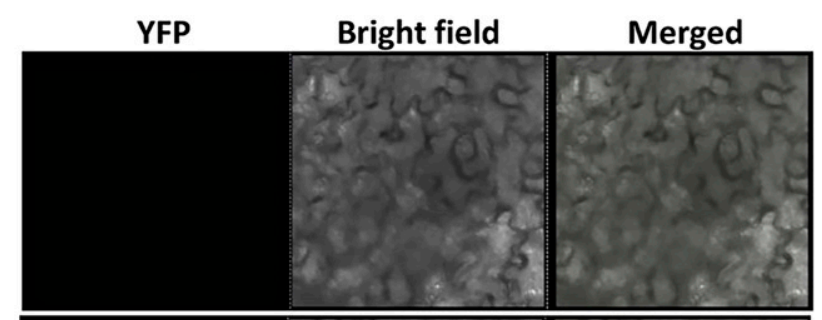

Clxyn24-nYFP
+
cYFP
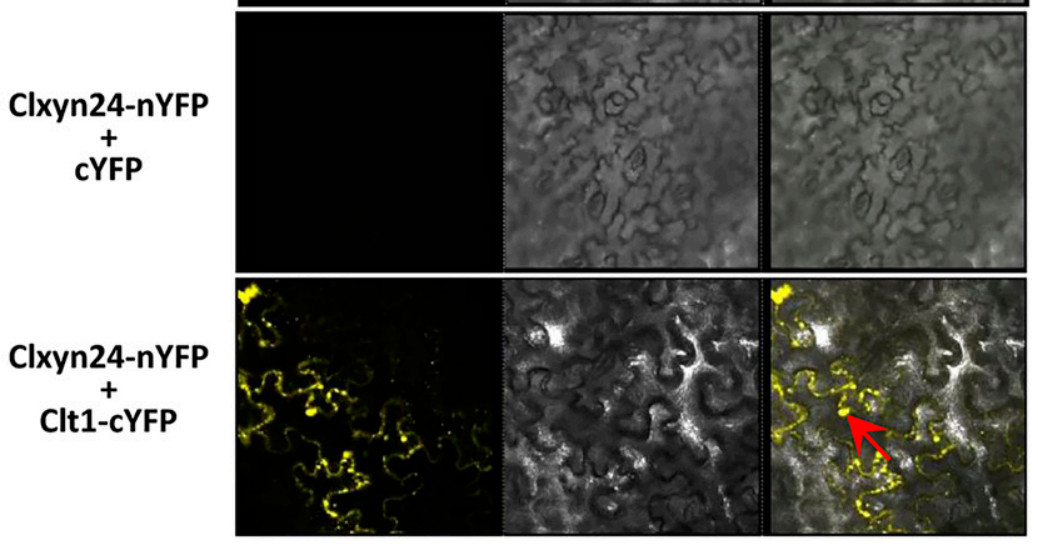

Fig. 1. The interaction between Clt1 and Clxyn24. A, Clt1 interacts with Clxyn24 in a yeast two-hybrid approach. The cell suspensions of Saccharomyces cerevisiae AH109 containing the indicated vectors were dropped onto the following synthetically defined (SD) media: SD-LT (SD without leucine and tryptophan), SD-LTHA/X (without leucine, tryptophan, histidine, and adenine supplemented with X- $\alpha$-Gal). Plasmid pairs pGADT7-SV40/pGBKT7Lam and pGADT7-SV40/pGBKT7-53 served as the negative and positive controls, respectively. B, Interaction analysis between Clt1 and Clxyn24 versions by bimolecular fluorescence complementation assay in infiltrated Nicotiana benthamiana leaves. Coexpression of Clt1 and Clxyn24 versions fused to split yellow fluorescent protein (YFP). n/cYFP = n- or c-terminal part of split YFP. Aggregates of Clt1 and Clxyn24 are indicated by an arrow. 
characteristic of xylanase (Supplementary Fig. S1B). Phylogenetic analysis showed that the Claxe43 protein falls in a dothideomycete group of acetyl xylan esterase homologs (Supplementary Fig. S2A). Claxe43 has 79\% identity with StAxe (Supplementary Fig. S2B). Alignment of acetyl xylan esterase homologs from C. lunata and S. turcica identified a conserved polysaccharide deacetylase domain, characteristic of acetyl xylan esterase (Supplementary Fig. S2B). The xylanase and esterase of S. turcica degrade xylan in the plant cell wall causing loss of integrity and aiding penetration (Condon et al. 2013; Degefu et al. 1997). We expect Clxyn24 and Claxe43 have a similar function in xylan metabolism.
Interactions of Clt1 with Clxyn24 and Claxe43.

To validate the interactions of Clt1 with Clxyn24 and Claxe43, we performed targeted $\mathrm{Y} 2 \mathrm{H}$ and bimolecular fluorescence complementation (BiFC) assays. The $\mathrm{Y} 2 \mathrm{H}$ results showed that the full length of Clt1 physically interacted with Clxyn24 and Claxe43 (Figs. 1A and 2A). In addition, the BiFC assay indicated that the interaction of Clt1 and Claxe43 form aggregates in the cytoplasm of the tobacco cells (Fig. 2B), while the interaction Clt1 and Clxyn24 could form aggregates in the cytoplasm and the area near the cell membrane (Fig. 1B). We speculate that the Clt1-Clxyn24 interaction broke the xylan into small-molecule oligosaccharides near the cell membrane, which could be further

A
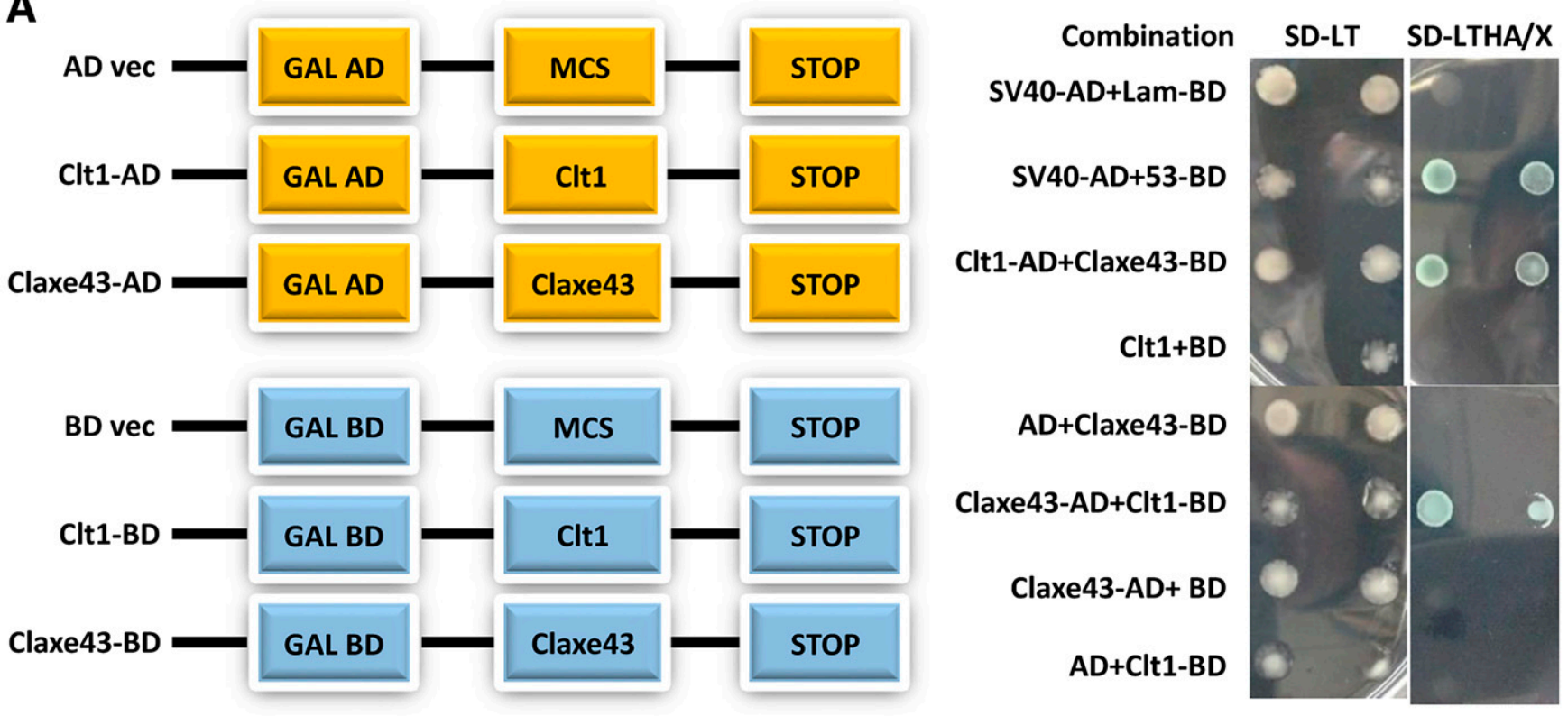

B

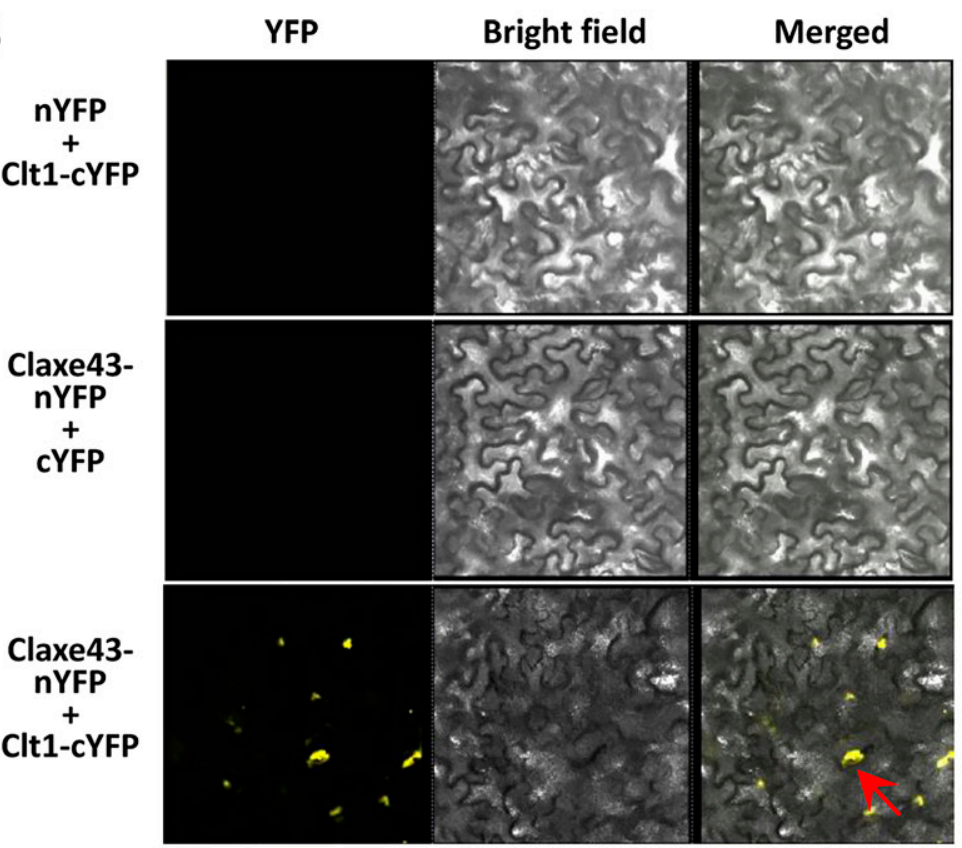

Fig. 2. The interaction between Clt1 and Claxe43. A, Clt1 interacts with Claxe43 in a yeast two-hybrid approach. The cell suspensions of Saccharomyces cerevisiae AH109 containing the indicated vectors were dropped onto the SD-LT (synthetically defined media without leucine and tryptophan) and SD-LTHD $+\mathrm{X}-\alpha-\mathrm{Gal}$ (SD without leucine, tryptophan, histidine, and adenine supplemented with X- $\alpha$-Gal). Plasmid pairs pGADT7-SV40/pGBKT7-Lam and pGADT7-SV40/pGBKT7-53 served as the negative and positive controls, respectively. B, Interaction analysis between Clt1 and Claxe43 versions by bimolecular fluorescence complementation assay in infiltrated Nicotiana benthamiana leaves. Coexpression of Clt1 and Claxe43 versions fused to split yellow fluorescent protein (YFP). n/cYFP: n- or c-terminal part of split YFP. Aggregates of Clt1 and Clxyn24 are indicated by an arrow. 
degraded into xylose by the combination of Clt1, Clxyn24, and Claxe43. The fused fragment containing cDNAs of CLT1 and green fluorescent protein was transformed in $C$. lunata $\mathrm{CX}-3$ to determine the subcellar location of Clt1. Results showed that Clt1 protein is localized in the cytoplasm (Supplementary Fig. S3), consistent with the phenomenon that Clt1 interacted with Clxyn24 and Claxe43 in the cytoplasm of the tobacco cells.

Identification of the interacting domain of Clt1 with Clxyn24 and Claxe43 in yeast cells.

Previous studies also indicated that the BTB domain is a protein-protein interaction motif, suggesting that the Clt1 may also interact with the $\mathrm{ClXyn} 24$ and $\mathrm{ClAxe} 43$ proteins. To further investigate whether the Clt1 interacts Clxyn24 and Claxe43 through its BTB domain, we removed the BTB domain, which was between the 169 and 265 amino acids from the Clt1 protein (abbreviated Clt1- $\triangle \mathrm{BTB}$ ). Then, we performed another $\mathrm{Y} 2 \mathrm{H}$ experiment with bait constructs expressing the GAL4 DNAbinding domain fused to the full-length Clxyn24 or Claxe43 protein and a series of prey constructs expressing the GAL4 transcriptional activation domain fused to full-length Clt1 (Clt1, 745 aa) or Clt1- $\Delta$ BTB (Clt1 $\Delta 169-265$ aa). As expected, no interaction of Clt1- $\Delta$ BTB with Clxyn24 and Claxe43 was detected (Fig. 3). The results indicated that the BTB domain of Clt1 was required for the interaction with Clxyn24 and Claxe43.

Involvement of Clxyn24 and Claxe43 on xylan utilization.

To research the functions of Clxyn24 and Claxe43, the target gene was replaced with a hygromycin resistance cassette. $\Delta$ clxyn24\&claxe 43 is a double mutant in which both the clxyn 24 and claxe43 genes were deleted (Supplementary Fig. S4). The strains were grown on solid medium, using xylan powder as the sole carbon source to test the effects of the deletion mutants on xylan utilization (Fig. 4A). The $\Delta$ clt1, $\Delta$ clxyn 24 , $\Delta$ claxe 43 , and $\Delta$ clxyn24\&claxe43 mutants did not exhibit significant xylan utilization compared with the wild-type (WT) strain. When we replaced xylan with xylose as the carbon source, the growth rate of $\Delta$ cltl significantly increased and $\Delta$ clxyn $24 \&$ claxe 43 had a slightly increased growth. However, the growth rate of $\Delta$ clxyn 24 and $\Delta$ claxe 43 was similar to those cultured on xylan medium (Table 1). In addition, the xylan powder utilization capacity of mutants also decreased in liquid medium (Fig. 4A; Table 1). The xylose reductase, xylitol dehydrogenase, and xylulokinase genes, which are related to xylose utilization, showed a similar expression pattern in the mutants compared with WT (Fig. 4B). The xylan utilization ability of the mutants was further tested by analyzing xylanase and acetyl xylan esterase production from the liquid culture. The activity of xylanase and the carbohydrate esterase were significantly decreased in these mutants (Fig. 4C). These results illustrated that Clxyn24 and Claxe43 were related to xylan utilization.

Having determined that Clt1 physically interacts with Clxyn24 and Claxe43 via its BTB domain, the next question is whether Clxyn24 and Claxe43 degrade xylan in a combinative manner with the help of Clt1. Therefore, we expressed and purified the Clt1, Clxyn24, and Claxe43 proteins and determined xylose production from xylan, using different protein combinations. More xylose was released when xylan was treated with Clxyn24\&Claxe43 compared with using Clxyn24 alone. However, no xylose was released when xylan was treated with Claxe43 or Clt1, separately. Interestingly, the xylose released amounts were increased when Clxyn24\&Claxe43 or Clxyn24 were used in combination with Clt1. We speculate that Claxe43 facilitates Clxyn24 attacking the main chains of xylan by eliminating the acetyl groups of xylan side chains, and Clt1 can enhance the activities of both proteins. However, neither Claxe43 nor Clt1 can degrade xylan by themselves directly (Fig. 4D). Overall, these results indicate that the interaction of Clt1 with Clxyn24 and Claxe43 facilitates the degradation of xylan, further providing a carbon source for $C$. lunata growth.

\section{Effects of Clxyn24 and Claxe43 on C. lunata conidiation.}

Preliminary characterization of $\Delta c l t 1$ revealed that Clt1 is responsible for $C$. lunata conidiation. Therefore, detecting the conidia production in Clxyn24 and Claxe43 mutants is significant for confirming both gene roles in the asexual reproduction via Clt1 regulation. $\Delta$ clxyn 24 and $\Delta$ claxe 43 produced fewer conidia on xylan plate compared with WT, while $\Delta$ clt1 and $\Delta$ clxyn $24 \&$ claxe43 showed the least conidiation ability. However, all strains produced more conidia on the xylose plate, and the difference of conidiation was not significant (Table 1).
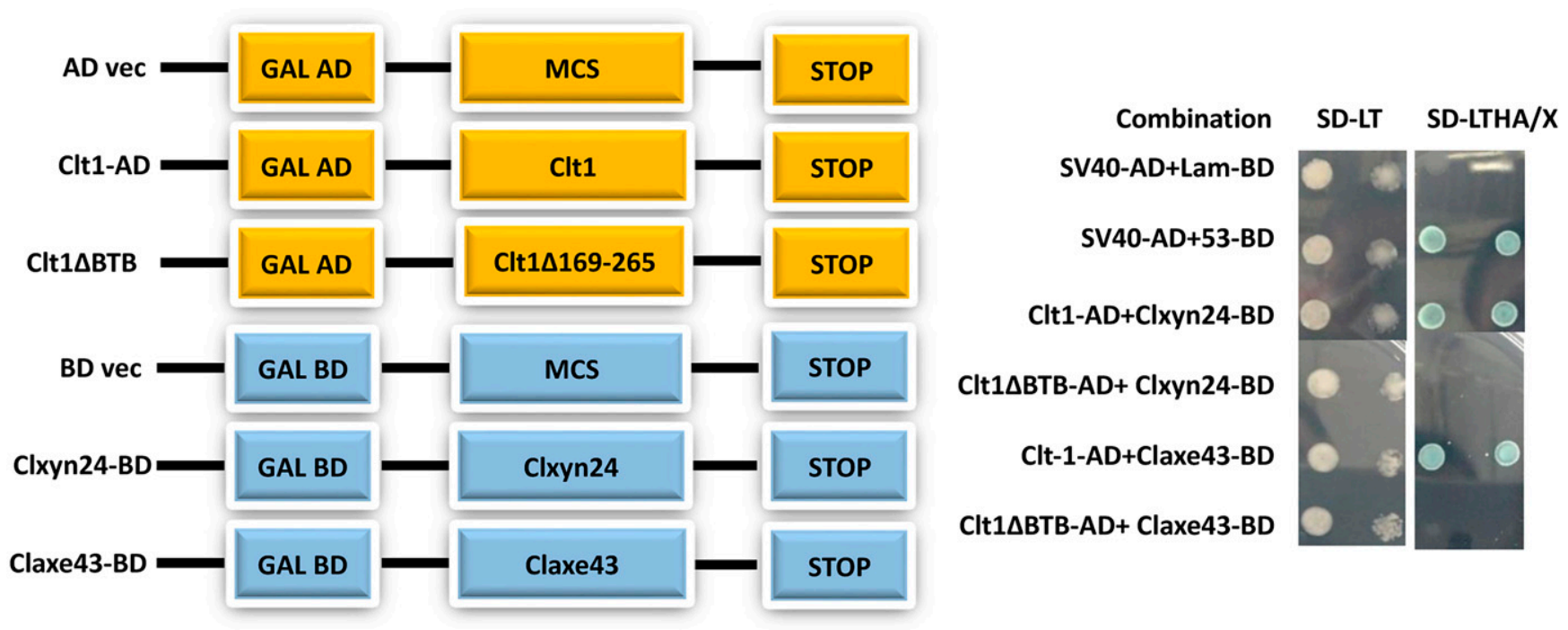

Fig. 3. Interacted motif identification of Clt1 with Clxyn24 and Claxe43. Yeast two-hybrid tests using full-length Clxyn24 (or Claxe43) and native (or variant) Clt1 fragments. SD-LT (synthetically defined medium without leucine and tryptophan) was used as nonselective media. SD-LTHA+X- $\alpha$-Gal (SD without leucine, tryptophan, histidine, and adenine supplemented with $\mathrm{X}-\alpha-\mathrm{Gal}$ ) was used as selective media. The assay displayed no interaction of Clt1- $\triangle$ BTB (Clt1 $\Delta 371-745)$ with Clxyn24 and Claxe43. Plasmid pairs of pGADT7-SV40/pGBKT7-53 and pGADT7-SV40/pGBKT7-Lam served as the positive and negative controls, respectively. 
Clxyn24 and Claxe43 are involved in the hyphal melanization.

Cultured both on solid (Fig. 4A) and in liquid xylan (Figs. 4A and 5A) medium, the pigmentation of $\Delta$ clxyn $24, \Delta$ claxe 43 , and $\Delta$ clxyn $24 \&$ claxe 43 cultures was not as dark as the WT but, also, not as light as $\Delta c l t 1$. When grown on solid xylose medium, colony color of $\Delta$ clxyn $24, \Delta$ claxe 43 , and $\Delta$ clxyn $24 \&$ claxe 43 turned dark green like WT, whereas the color was just partially restored in $\Delta c l t 1$ (Fig. 4A). Results indicated that Clxyn24 and Claxe 43 were involved in the melanization of mycelia when cultured using xylan powder as the sole carbon source. To verify

A

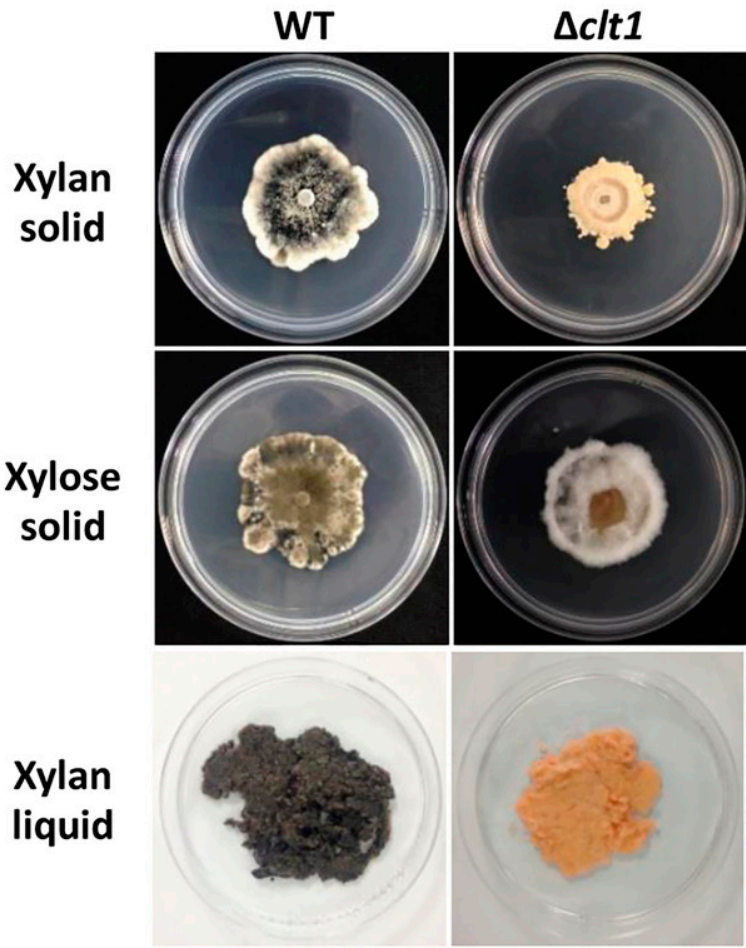

$\Delta c / x y n 24$

$\Delta c l a x e 43 \quad \Delta c l x y n 24 \&$ claxe43
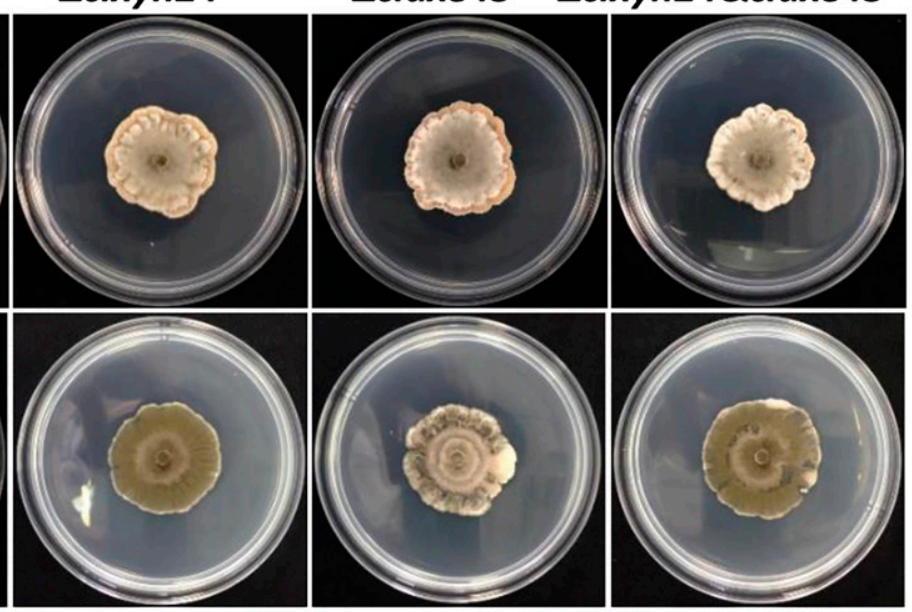

B
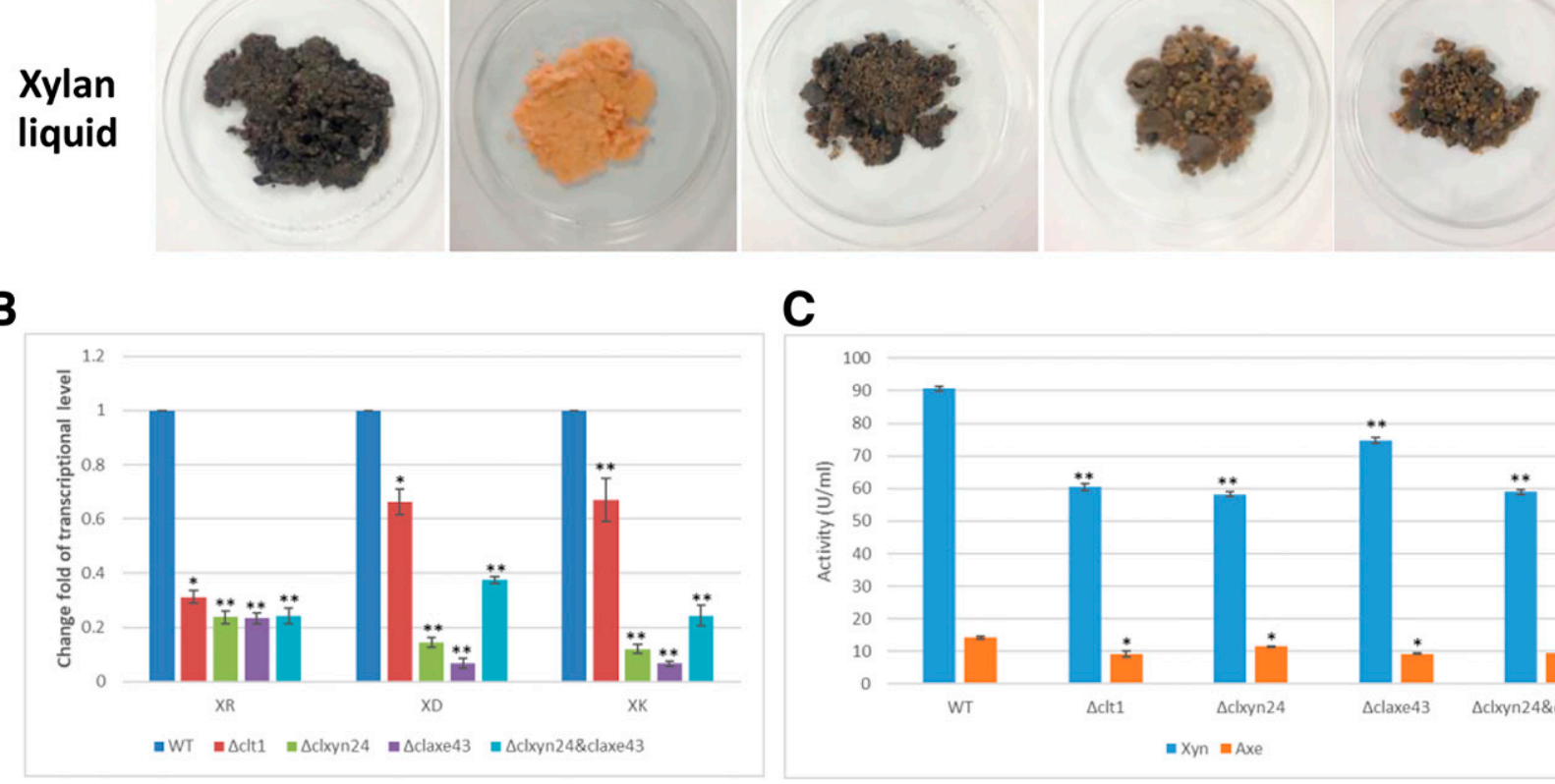

C

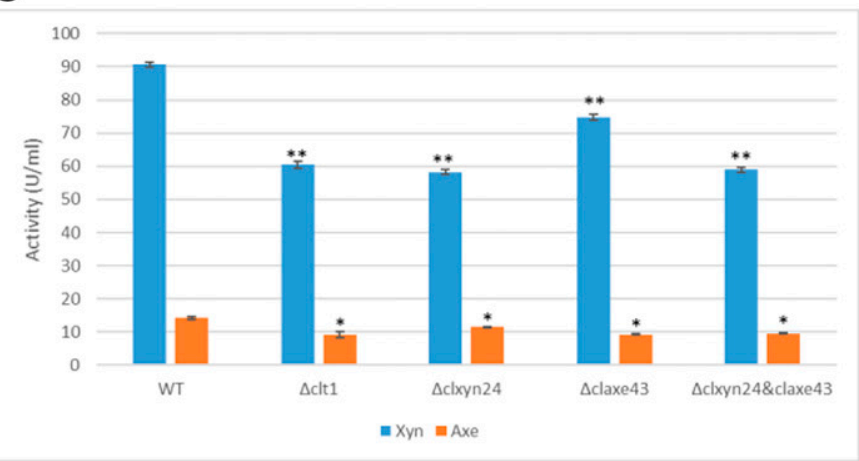

D

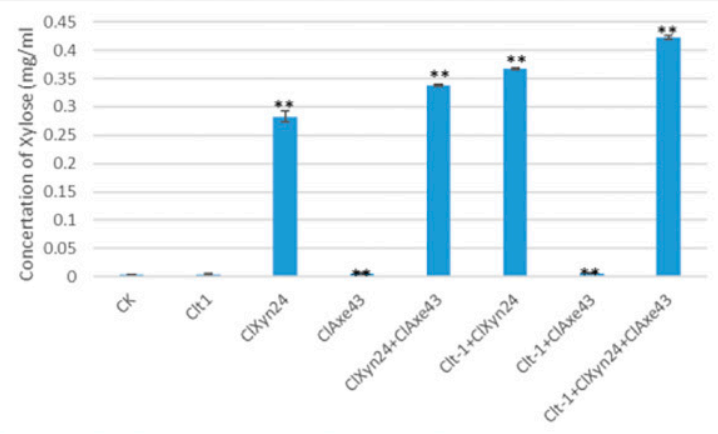

Fig. 4. Growth and xylan utilization of the deletion mutant and wild-type (WT) strains. A, Growth of the mutant and WT strains on solid medium with xylan or xylose powder as the carbon source and in liquid medium with xylan powder as the carbon source. B, Quantitative reverse transcription (qRT)PCR analysis of xylose utilization gene expression levels in mutants compared with WT. XR $=$ xylose reductase, $\mathrm{XD}=\mathrm{xylitol}$ dehydrogenase, and $\mathrm{XK}=$ xylulokinase. $\mathbf{C}$, Enzyme activity test of mutant and WT strains. Xyn $=$ xylanase and Axe = carbohydrate esterase. D, Effects of different proteins on the release of xylose. The bars indicate the standard errors of the three repeated trials. A single asterisk indicates $P$ value $<0.05$, while double asterisks indicate $P$ value $<0.001$ in the T-test analysis. $\Delta$ clt $1=$ clt1 mutant, $\Delta$ clxyn $24=$ clxyn 24 mutant, $\Delta$ claxe $43=$ claxe 43 mutant, and $\Delta$ clxyn $24 \&$ claxe $43=$ clxyn 24 and claxe 43 double mutant. 
this observation, we analyzed the relative mRNA expression of the PKS18, CMR1, BRN1, BRN2, and SCD genes associated with melanin synthesis in the mutants and WT. Quantitative reverse transcription (qRT)-PCR analyses indicated that the expression levels of PKS18 decreased in all mutants compared with WT. It was found that the expression of PKS18 had a 16.44-fold decrease in $\Delta c l t 1$ and a 22.03 -fold decrease in $\Delta$ clxyn $24 \&$ claxe 43. $C M R 1, B R N 1, B R N 2$, and $S C D$ also showed low expression patterns in mutants (Fig. 5B). Overall, we conclude that ClXyn24 and $\mathrm{ClAxe} 43$ as well as Clt1 affect the pigmentation in $C$. lunata.

\section{Clxyn24 and Claxe 43 are essential}

for M5HF2C toxin biosynthesis and virulence in $C$. lunata.

Reports indicate that Clt1 regulates the synthesis of M5HF2C toxin. Therefore, detecting the M5HF2C toxin production in ClXyn24 and ClAxe43 mutants is necessary. After culturing in Fries 3 medium with xylan powder as the carbon source for 30 days, the amount of M5HF2C toxin produced by $\Delta$ clxyn 24 and $\Delta$ claxe 43 showed a sharp decline as compared with WT. When both genes were deleted simultaneously, the M5HF2C production of the mutant were $90.80 \%$ lower than that produced by WT (Fig. 6). However, the capabilities of M5HF2C production were increased in all strains when using xylose powder instead of xylan, and the difference between the mutants and WT declined. As shown in Figure 5B, the expression of PKS18 and $B R N 1$ genes, which are associated with M5HF2C biosynthesis, fell sharply in the mutants (Liu et al. 2011). The experiment results indicated that Clxyn24 and Claxe43 affected the production of M5HF2C toxin. More interestingly, no M5HF2C was detected in $\Delta$ clt1 grown in either xylan or xylose Fries 3 medium. We speculated that Clt1 might directly regulate other unknown proteins responsible for M5HF2C synthesis.

Because of the decreased ability to produce M5HF2C, we further tested the pathogenicity of mutants, using inoculation on detached maize leaves. The lesion area size showed a decreasing trend from WT to $\Delta$ claxe $43, \Delta$ clxyn $24, \Delta$ clxyn $24 \&$ claxe 43 , and $\Delta c l t 1$ (Fig. 7; Table 1), indicating that ClXyn24 and ClAxe43 as well as Clt1 were required for the whole virulence in $C$. lunata.
A

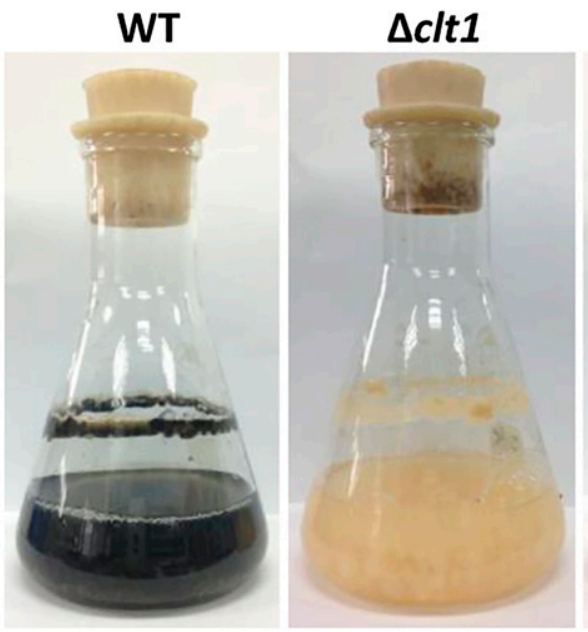

$\Delta c \mid x y n 24$

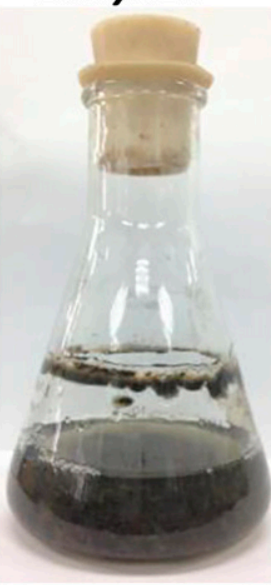

$\Delta$ claxe43 $\Delta c 1 x y n 24 \&$ claxe43

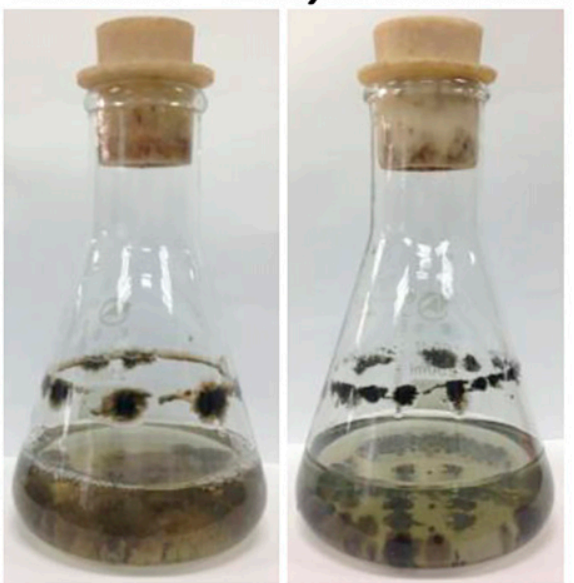

B

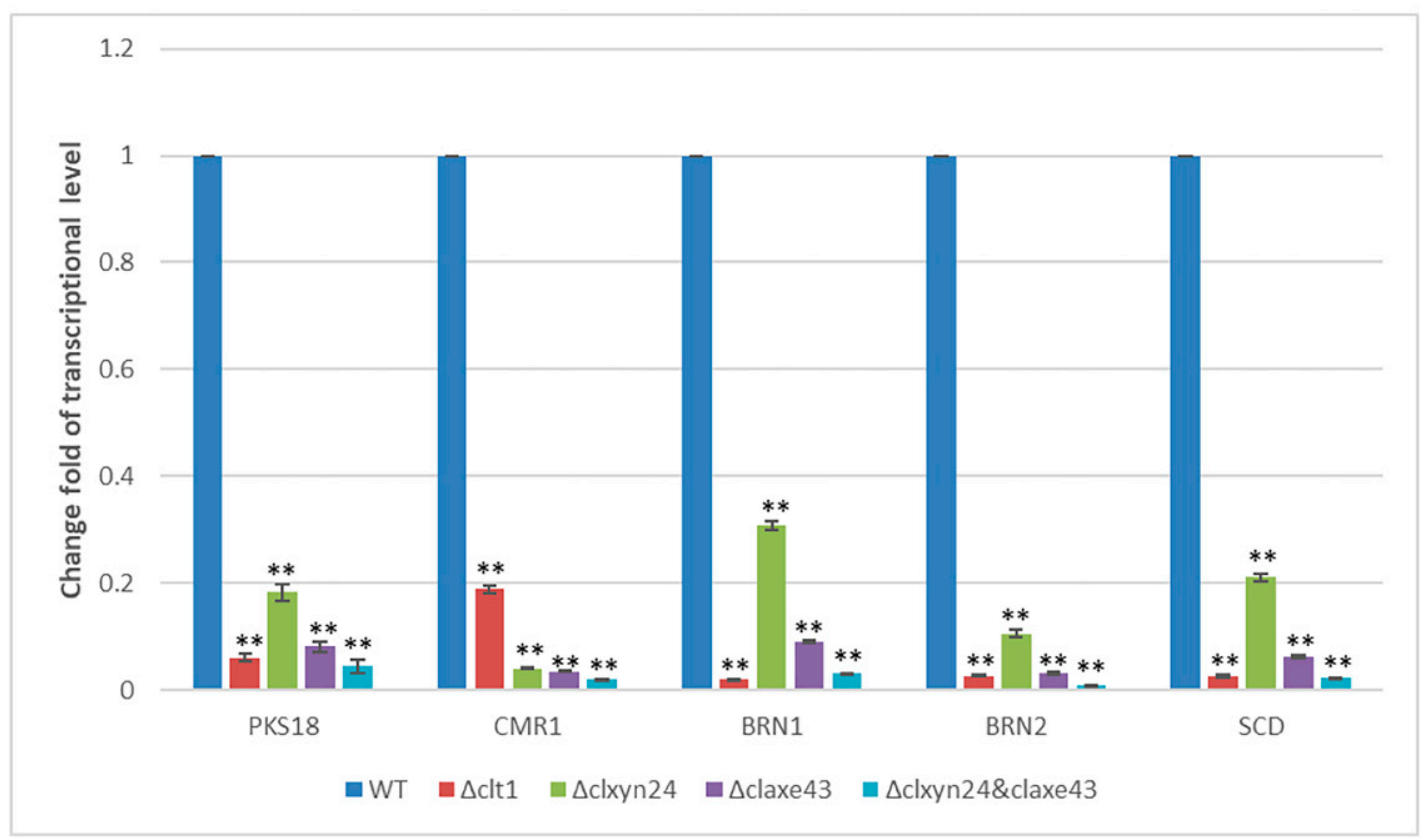

Fig. 5. Clxyn 24 and Claxe 43 are involved in the melanization of mycelia. A, Growth of the mutant and wild-type (WT) strains in liquid medium with xylan as the carbon source. Note the light melanization of mycelia of mutants compared with WT. B, Quantitative reverse transcription (qRT)-PCR analyses of PKS18, CMR1, BRN1, BRN2, and SCD. Expression levels compared with the WT are shown. Error bars are the standard deviation. Double asterisks indicate the $P$ value $<0.001$ in a T-test analysis. WT, wild-type strain; $\Delta$ clt1, clt1 mutant; $\Delta$ clxyn 24 , clxyn 24 mutant; $\Delta$ claxe 43 , claxe 43 mutant; $\Delta$ clxyn $24 \&$ claxe 43, clxyn 24 and claxe 43 double mutant. 
Acetyl-CoA derived from xylan degradation is utilized for toxin and melanin precursor synthesis.

PKS18 was functionally characterized to encode a PKS that converts malonyl CoA to 1,3,6,8-tetradroxynaphthalene $(1,3,6,8$ $\mathrm{THN}$ ) in the DHN-melanin synthesis pathway (Eliahu et al.
2007). Like other toxins, M5HF2C toxin is also derived from a polyketide intermediate that is synthesized by PKS18 (Baker et al. 2006). As the melanin and toxin biosynthetic pathway begins from malonyl-CoA, which is derived from acetyl-CoA, acetyl-CoA derived from xylan degradation is considered

Table 1. Phenotypic analysis of Curvularia lunata mutants compared with wild-type isolate CX-3 $3^{\mathrm{v}}$

\begin{tabular}{|c|c|c|c|c|c|c|}
\hline \multirow[b]{2}{*}{ Strain } & \multicolumn{2}{|c|}{ Solid mediumw } & \multirow{2}{*}{$\begin{array}{c}\text { Liquid medium } \\
\text { Xylan }^{\mathrm{x}}\end{array}$} & \multicolumn{2}{|c|}{ Conidiation $^{\mathrm{y}}$} & \multirow[b]{2}{*}{ Necrosis $\operatorname{area}^{z}$} \\
\hline & Xylan & Xylose & & Xylose & Xylan & \\
\hline Wild type & $4.81 \pm 0.14 \mathrm{a}$ & $4.79 \pm 0.14 \mathrm{a}$ & $6.46 \pm 0.19 a$ & $5.57 \pm 0.05 a$ & $6.25 \pm 0.08 \mathrm{a}$ & $70.17 \pm 2.65 \mathrm{a}$ \\
\hline$\Delta$ clt1 & $2.83 \pm 0.21 \mathrm{c}$ & $3.93 \pm 0.28 b$ & $3.79 \pm 0.05 b c$ & $4.80 \pm 0.08 \mathrm{~d}$ & $5.44 \pm 0.06 c$ & $21.50 \pm 1.22 \mathrm{~cd}$ \\
\hline$\Delta$ clxyn 24 & $3.99 \pm 0.15 b$ & $3.84 \pm 0.10 \mathrm{~b}$ & $4.15 \pm 0.03 b$ & $5.26 \pm 0.11 \mathrm{ab}$ & $5.80 \pm 0.06 b$ & $38.00 \pm 2.94 b$ \\
\hline$\Delta$ claxe 43 & $3.82 \pm 0.05 b$ & $3.81 \pm 0.07 b$ & $4.31 \pm 0.21 b$ & $5.16 \pm 0.07 b c$ & $5.67 \pm 0.09 b c$ & $26.67 \pm 1.09 c$ \\
\hline$\Delta$ clxyn $24 \&$ claxe 43 & $3.60 \pm 0.03 b$ & $3.92 \pm 0.16 b$ & $3.39 \pm 0.16 c$ & $4.90 \pm 0.08 \mathrm{~cd}$ & $5.66 \pm 0.06 b c$ & $15.17 \pm 1.34 d$ \\
\hline
\end{tabular}

${ }^{v}$ Data in all columns are the means of three independent experiments with standard deviation. The statistical analysis was conducted using the SAS statistical package. Statistically significant analysis of variance was analyzed using least significant difference tests. Different letters in each data column indicate significant differences at $P=0.05$.

${ }^{\mathrm{w}}$ Diameter (in millimeters) of hyphal radii at day 21 after incubation on solid medium with xylan or xylose powder as the carbon source at $28^{\circ} \mathrm{C}$.

${ }^{x}$ Dry weight of mycelia (in grams) at day 9 after incubation in liquid medium with xylan as the carbon source with continuous shaking at $180 \mathrm{rpm}$ at $28^{\circ} \mathrm{C}$.

y Conidial numbers (log transformation) shown as $\log _{10}$ of colony-forming units per milliliter.

${ }^{\mathrm{z}}$ Necrosis area of detached leaves inoculation shown in square millimeters.

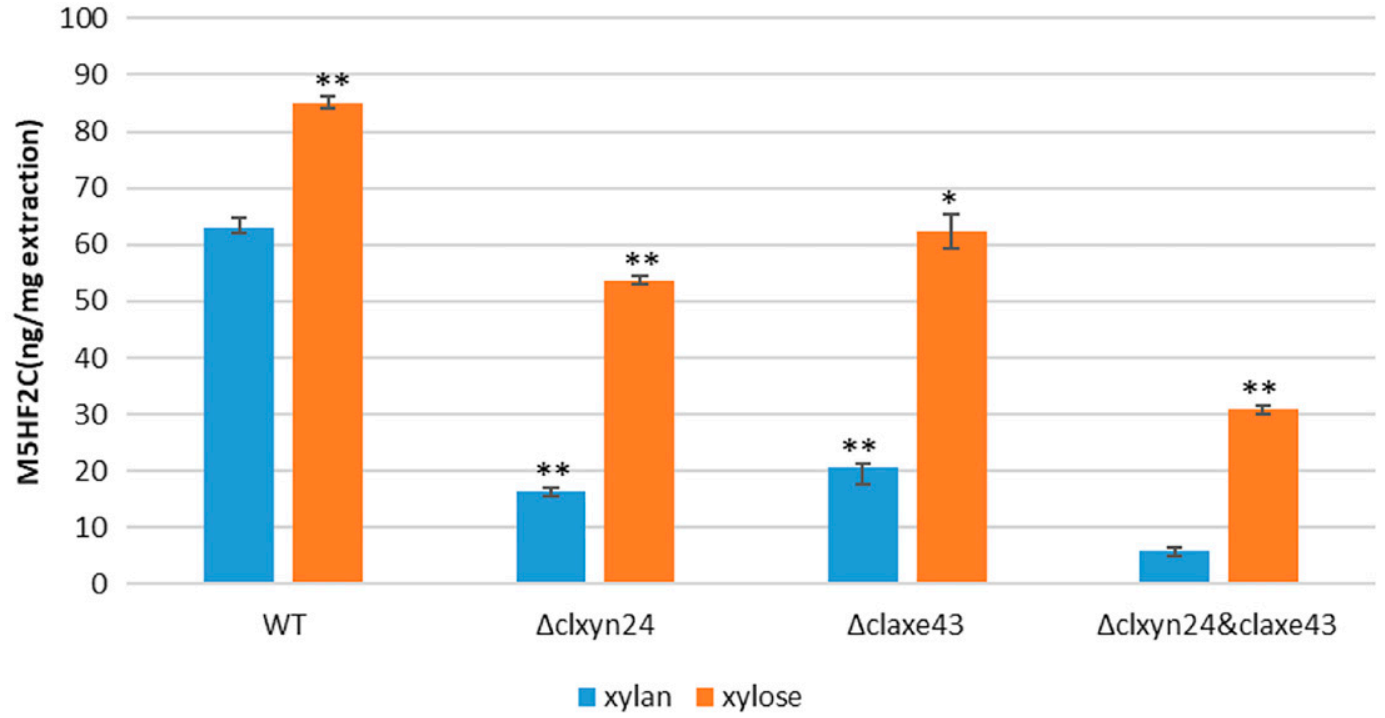

Fig. 6. Clxyn24 and Claxe43 regulate the biosynthesis of M5HF2C toxin. Amount of M5HF2C (per mg extraction) produced by mutants and wild-type (WT) strain that were cultured in Fries 3 medium with xylan or xylose as the carbon source after 30 days. The bars indicate the standard errors of the three repeated trials. A single asterisk indicates the $P$ value $<0.05$ while double asterisks indicate the $P$ value $<0.001$ in the T-test analysis. $\Delta c l t 1=$ clt 1 mutant, $\Delta$ clxyn $24=$ clxyn 24 mutant, $\Delta$ claxe $43=$ claxe 43 mutant, and $\Delta$ clxyn $24 \&$ claxe $43=$ clxyn 24 and claxe 43 double mutant.
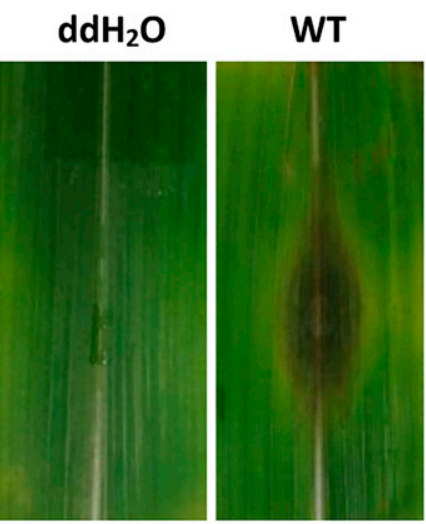

$\Delta c l t 1$

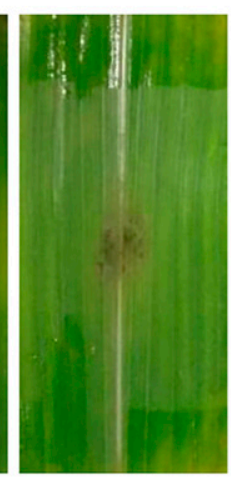

\section{$\Delta c l x y n 24$}

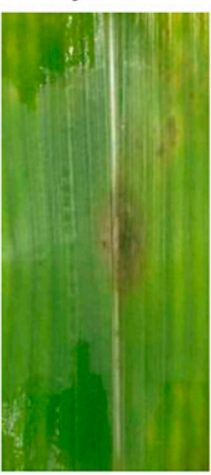

$\Delta$ claxe43 $\Delta$ clxyn24\&claxe43
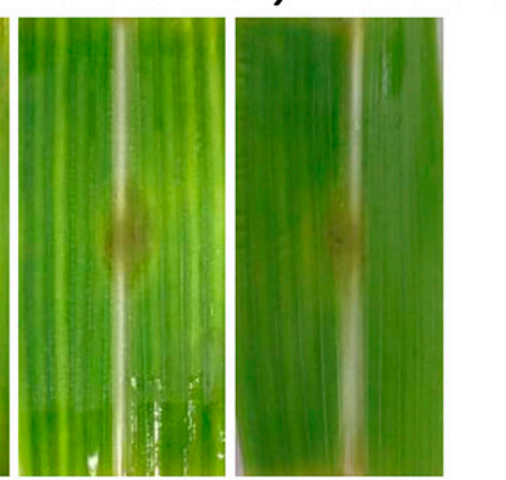

Fig. 7. Virulence of mutants and wild-type (WT) strain on maize leaves. $\Delta$ clt1, $\Delta$ clxyn $24, \Delta$ claxe 43 , and $\Delta$ clxyn $24 \&$ claxe 43 are impaired in the colonization of maize leaves. Detached leaves of HUANGZAO-4 were inoculated with conidial suspensions and were incubated on two layers of filter papers moisturized with $10 \mathrm{mM}$ 6-benzyladenine (6-BA) in Petri dishes at $28^{\circ} \mathrm{C}$ for $96 \mathrm{~h} . \Delta$ clt $1=$ clt 1 mutant, $\Delta$ clxyn $24=$ clxyn 24 mutant, $\Delta$ claxe $43=$ claxe 43 mutant, and $\Delta$ clxyn $24 \&$ claxe $43=$ clxyn 24 and claxe 43 double mutant. 
required for the synthesis of melanin and toxin in C. lunata. To assess this possibility, we detected the acetyl-CoA and malonylCoA content of mutants and WT strains. Results indicated that $\Delta$ clt1, $\Delta$ clxyn $24, \Delta$ claxe 43 , and $\Delta$ clxyn $24 \&$ claxe 43 produced less acetyl-CoA and malonyl-CoA than the WT (Fig. 8A). Studies have shown that lower utilization of xylan leads to lower accumulation of acetyl-CoA and malonyl-CoA in mutants. Furthermore, we tested the expressions of the PDE1A (pyruvate dehydrogenase alpha subunit), $P D E 1 B$ (pyruvate dehydrogenase beta subunit), $P D X$ (pyruvate dehydrogenase protein $\mathrm{X}$ ), and $D D$ (dihydrolipoyl dehydrogenase) genes responsible for acetylCoA synthase and the ACC (acetyl-CoA carboxylase) gene responsible for acetyl-CoA synthase in the mutants. The expression levels of PDE1A, PDE1B, PDX, DD, and $A C C$ genes were lower in mutants compared with WT, which is consistent with the phenotype that mutants exhibited reduced accumulation of acetyl-CoA and malonyl-CoA (Fig. 8B).

Global exploration of $C L T 1$ regulated genes in $C$. lunata.

To reveal how CLT1 regulates melanin and toxin formation and pathogenicity in $C$. lunata, we performed transcriptome analysis by comparing the toxin production-deficient mutant (T806) and WT (CX-3), using RNA sequencing (Gao et al. 2014a). We identified a total of 5,446 expressed genes in at least three pairwise comparisons. This analysis yielded 4,524 differentially expressed genes, including 792 putative pathogen-host interaction (PHI) genes between T806 and CX-3 (2,118 of which were up-regulated and 2,406 were down-regulated). These results indicate that changes in gene expression in $C$. lunata caused by the disruption of clt1 were significant (false discovery rate [FDR] $\leq 0.05)$.

\section{A 60}
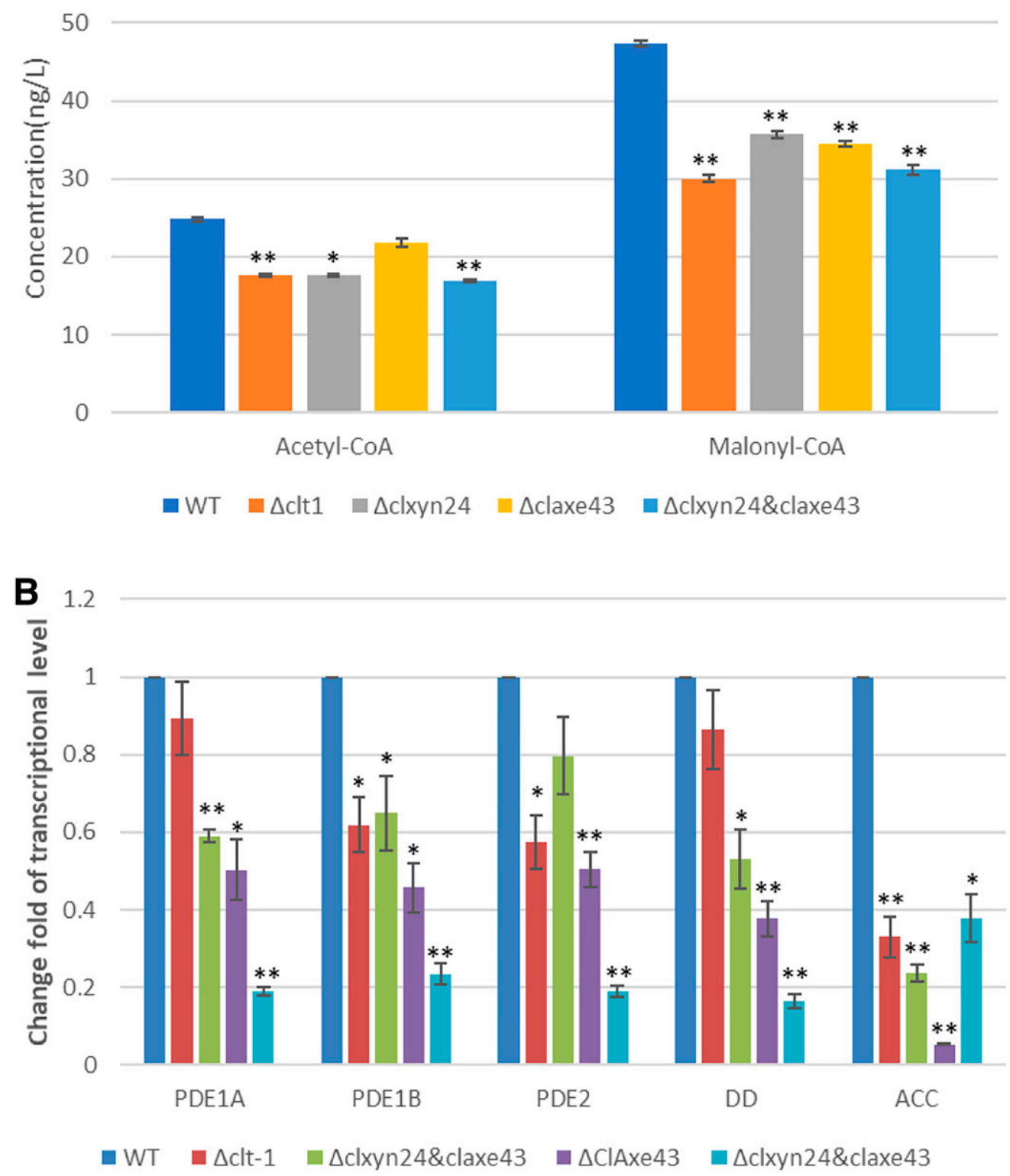

Fig. 8. Clxyn24 and Claxe43 regulate the acetyl-CoA and malonyl-CoA accumulation. A, Determination of acetyl-CoA and malonyl-CoA biosynthesis in mutants and wild-type (WT) strains. B, Quantitative reverse transcription (qRT)-PCR analysis of genes that are responsible for acetyl-CoA and malonylCoA biosynthesis. The bars indicate the standard errors of the three repeated trials. A single asterisk indicates $P$ value $<0.05$, while double asterisks indicate $P$ value $<0.001$ in the T-test analysis. $\Delta$ clt $1=$ clt 1 mutant, $\Delta$ clxyn $24=$ clxyn 24 mutant, $\Delta$ claxe $43=$ claxe 43 mutant, and $\Delta$ clxyn $24 \&$ claxe $43=$ clxyn 24 and claxe 43 double mutant. 


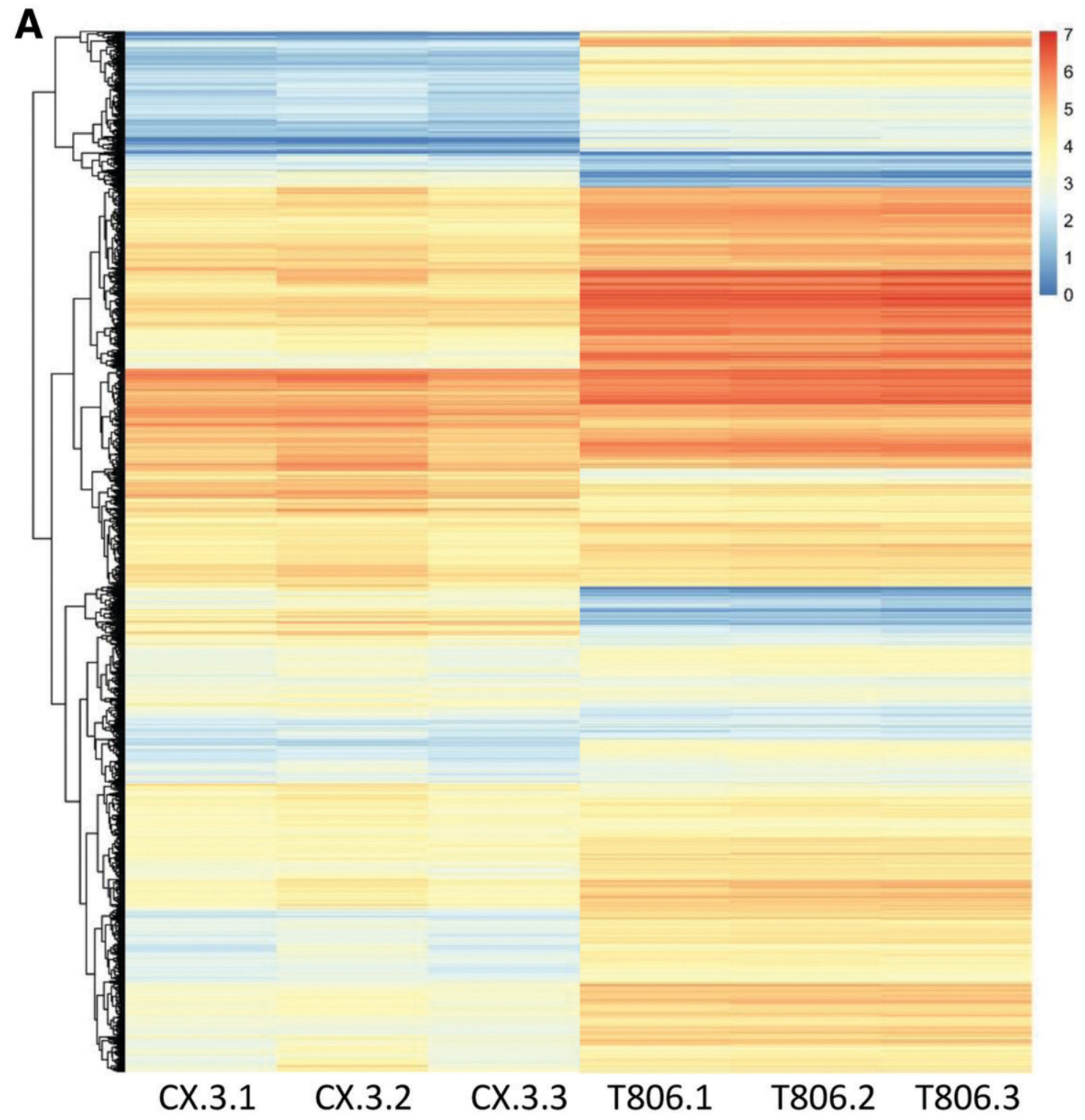

B

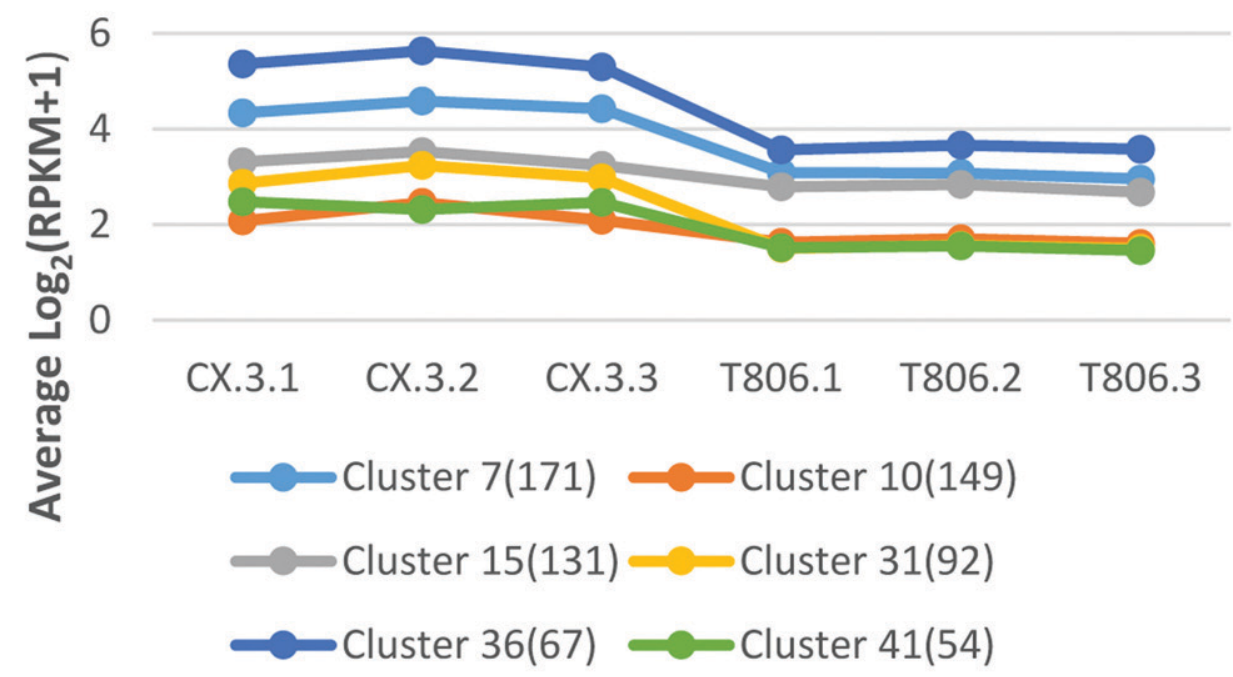

Fig. 9. Clustering of gene expression. A, The $\log _{2}$ reads per kilobase million (RPKM) for each gene was used for hierarchical analysis of the heat map for each of the selected strains (CX-3 and T806). The heat map illustrates that 5,446 expressed genes were classified into 43 gene-expression clusters generated by clustering. The colors correspond to the $\log _{2}$ RPKM values, ranging from bright blue to red. $\mathbf{B}$, $\log _{2}$ average gene-expression levels of the six downregulated clusters identified in T806 compared with CX-3. The number of genes in each cluster is bracketed. 
Clustering affinity search technique (CAST) of the 5,446 expressed genes revealed 43 clusters, with gene numbers ranging from 51 to 616 (Fig. 9A). Decreased transcript levels were observed in clusters $7,10,15,31,36$, and 41 in T806 as compared with CX-3, comprising $35.93 \%$ of the total expressed genes (Fig. 9B). The results of gene ontology enrichment analysis showed a lower representation of distinct gene functions in the above six clusters. Genes encoding proteins related to metabolic processes, such as metabolic process, protein processing, and protein maturation, were enriched in cluster 7 . The genes in cluster 10 function mainly in nucleoside metabolic process, and genes in clusters 15,31 , and 41 were functionally enriched in membrane formation. The functional enrichment of cluster 36 revealed disaccharide and oligosaccharide metabolic processes (Supplementary Table S2). A total of 14 different families were selected to analyze the expression patterns of $C$. lunata in growth and pathogenesis (Fig. 10). There were down-regulated genes encoding products involved in glycolysis and gluconeogenesis (Fig. 10A), histidine kinase phosphorelay (Fig. 10B), and bZIP transcription factors (Fig. 10C) as well as major facilitator superfamily transporters (Fig. 10D) in T806.

\section{Expression profiles of genes involved in melanin and toxin synthesis.}

Genes related to melanin production were searched in the C. lunata genome, including a PKS (PKS18, CL07170), tretrahydroxynaphthalene reductase (BRN2, CL04688), SCD (CL04685), and 1,3,8-naphthalenetriol reductase (BRN1, CL07173) (Keller and Hohn 1997). Expression levels of genes encoding these enzymes are shown in Figure 11A. CL07170 (PKS18), CL04688 (BRN2), CL04685 (BRN1), and CL07173 (SCD) homologs in T806 were all significantly down-regulated (over 2.5 -fold change) as compared with $\mathrm{CX}-3$, which is consistent with the phenotypic characteristic of melanin production Gao et al. 2014a and b; Gao and Chen 2017.
BRN1 (CL07173) is also related to the biosynthesis of M5HF2C toxin in C. lunata (Liu et al. 2011). More interesting, 13 flanking genes of BRN1 in C. lunata genome were down-regulated in T806, including PKS (CL07170), transcription factor BMR1 (CL07172), ribosome biogenesis protein (CL07174), exosome complex exonuclease (CL07178), nucleolar protein (CL07180), prenylcysteine oxidase (CL07181), cytochrome P450 (CL07182), translation initiation factor IF-2 (CL07184), ABC transporter (CL07186), and hypothetical protein (CL07176, CL07179, CL07183, CL07185) genes. BRN1 and its 13 flanking genes are found clustered in a region spanning $57.89 \mathrm{~kb}$ on scaffold 9 of the C. lunata CX-3 genome (Fig. 11B). Among the 14 genes, CL07170, CL07172, CL07173, and CL07186 were PHI genes. Notably, CL07170 encoding PKS18 was down-regulated in T806. Interestingly, a pks 18 deletion mutant exhibited the same phenotype as the brnl-silent mutant that cannot produce M5HF2C toxin and melanin and loses the infective ability in maize leaves (Gao and Chen 2017). Thus, we speculate that BRN1 and PKS18 are the key genes responsible for toxin synthesis, and this cluster is a putative toxin biosynthesis gene cluster in $C$. lunata.

\section{DISCUSSION}

Clt1 has a BTB domain, which is found highly conserved throughout eukaryotes as a protein-protein interaction motif (Perez-Torrado et al. 2006). The BTB domain performs different functions by combining with other domains in various organisms, such as protein degradation and gene transcriptional regulation (Perez-Torrado et al. 2006; Zollman et al. 1994). To elucidate the mechanism of Clt1 in controlling M5HF2C toxin production, the formation of melanin and conidiation and pathogenicity (Gao et al. 2014a), a C. lunata Y2H cDNA library was constructed and was used to screen interacting proteins of Clt1. Two screened proteins, Clxyn24 and Claxe43, were picked for bioinformatics analysis and gene function research.
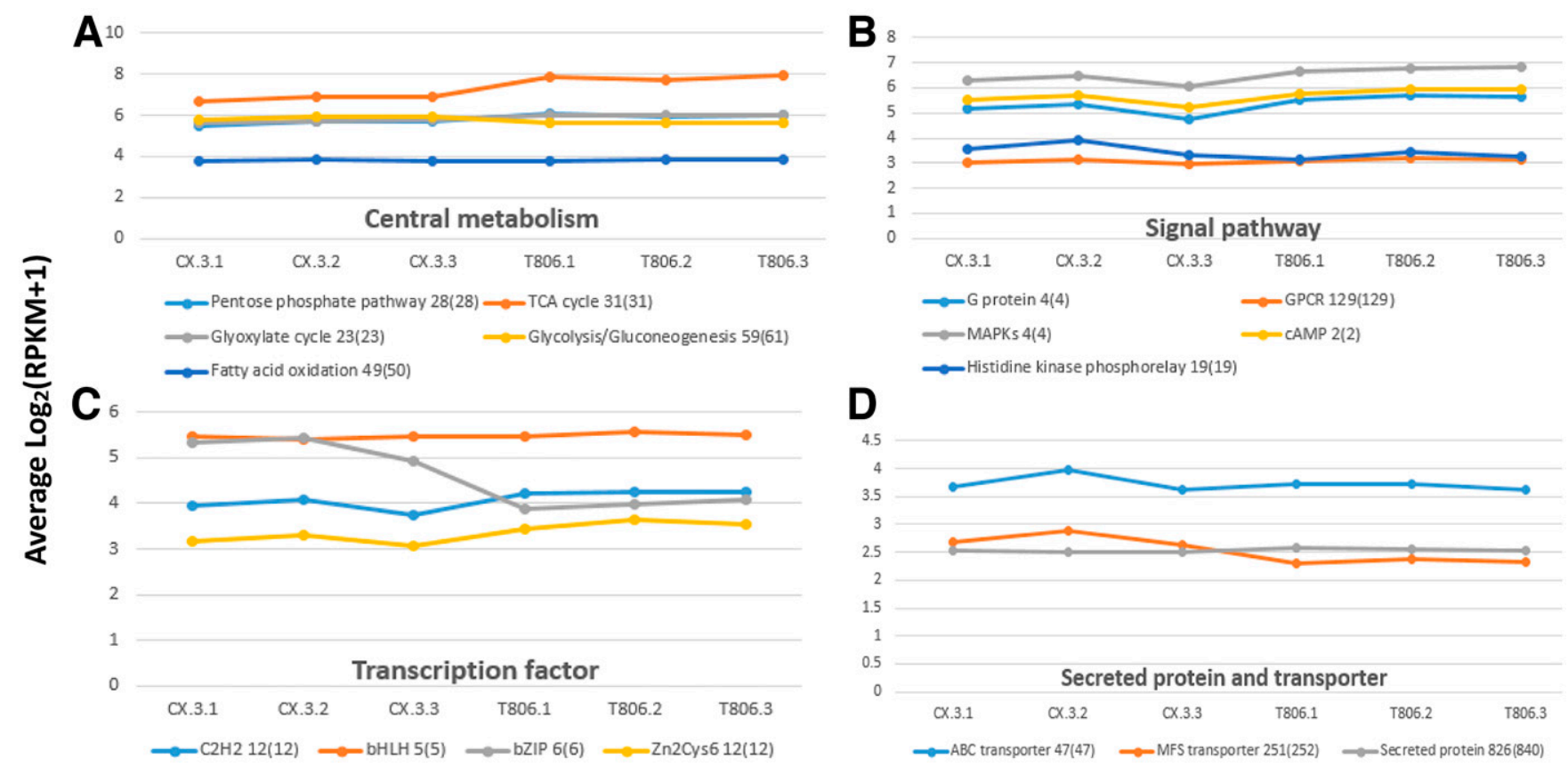

Fig. 10. Global expression analysis of genes encoding proteins in different functional categories. A, The central metabolic pathways include the pentose phosphate pathway, TCA cycle, glyoxylate cycle, glycolysis/gluconeogenesis, and fatty acid oxidation. B, Signal transduction and components, such as G protein, GPCR (G protein-coupled receptors), mitogen-activated protein kinase cascades, cAMP signaling, and histidine kinase phosphorelay signaling. C, Four main classes of transcription factors, i.e., C2H2, bHLH, bZIP, and Zn2Cys6. D, Putative secreted proteins, ABC transporter, and major facilitator superfamily (MFS) transporter genes. The $y$ axes are $\log _{2}$-transformed total intensities. The total numbers within a gene family are shown in numerals and the numbers of expressed genes within a family are shown in parentheses. 
Clxyn24 was identified as a homolog of xylanase from Cochliobolus sativus, and Claxe43 was identified as a homolog of acetyl xylan esterase from Bipolaris oryzae. $\mathrm{Y} 2 \mathrm{H}$ and $\mathrm{BiFC}$ assays were conducted to verify the interaction of Clt1 with full-length Clxyn24 (Fig. 1) and Claxe43 (Fig. 2). Furthermore, a Y2H study revealed that Clt1 physically interacted with Clxyn24 and Claxe43, through its BTB domain, to degrade xylan, which was used as a carbon source for $C$. lunata growth (Fig. 3). We also found that Clt1 protein is localized in the cytoplasm (Supplementary Fig. S3), consistent with the BiFC assay that Clt1 interacted with Clxyn24 and Claxe43 in the cytoplasm of tobacco cells.

To explore the functional relationship of Clt1 with Clxyn24 and Claxe43, we investigated the biological functions of all C. lunata mutants. In the pathogenicity tests, the mutants produced smaller lesions on inoculated maize leaves, which were similar to the phenotype of $\Delta c l t 1$ described above (Fig. 7). Clt1 protein has been reported to regulate melanin and toxin production in C. lunata (Gao et al. 2015b). The clt1 deletion mutant showed decreased M5HF2C toxin production and synthesized a light pigment (Gao et al. 2014a). In the current study, we observed that $\Delta$ claxe $43, \Delta$ clxyn 24 , and $\Delta$ clxyn $24 \&$ claxe 43 as well as $\Delta c l t 1$ produced significantly low levels of melanin (Figs. $4 \mathrm{~A}$ and $5 \mathrm{~A}$ ). Furthermore, the expression levels of DHN melanin biosynthesis genes were simultaneously down-regulated in all mutants (Fig. 5B). Meanwhile, the mutants produced a lesser amount of M5HF2C toxins (Fig. 6).

The CLT1 gene is closely related to the production of M5HF2C toxin and melanin in C. lunata (Gao et al. 2014a). The proteomic approach based on two-dimensional gel electrophoresis and mass spectrometry techniques identified another melanin biosynthesis protein, SCD, that is associated with Clt1 expression in $C$. lunata wild-type CX-3 and the clt1 deletion mutant $\Delta$ clt1 (Gao et al. 2015c). It is remarkable that the silencing of the 1,3,8-trihydroxynaphthalene (THN) reductase gene BRN1 (GenBank accession number DQ358052), which converts $1,3,8-\mathrm{THN}$ to DHN in the DHN melanin, also lost the capacity to produce the toxin (Liu et al. 2011). Thirteen genes flanking BRN1 (CL07173) were found, in the transcriptome analysis, to be lowly expressed in T806 (Fig. 11B). The toxin biosynthetic pathway involves many structural, secretory, and regulatory genes. As known, genes responsible for secondary metabolite biosynthesis were usually clustered (Fox et al. 2008; Inderbitzin et al. 2010). These genes are clustered in a region spanning
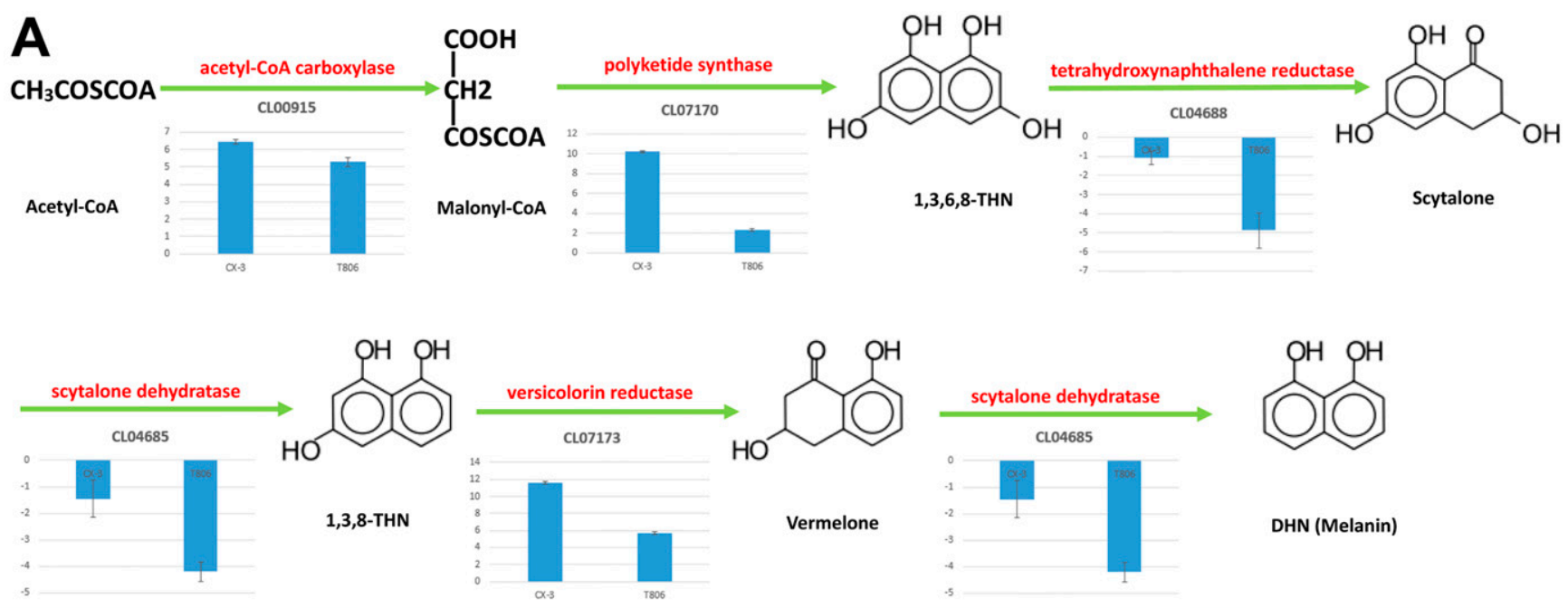

B

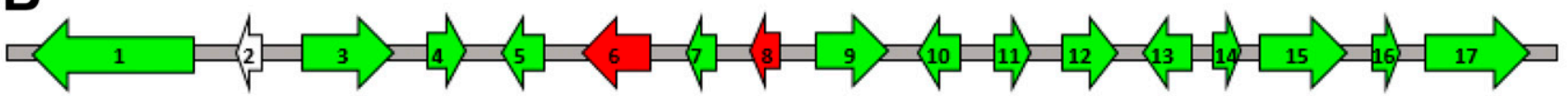

\section{1: Polyketide synthase (CL07170) \\ 2: Hypothetical protein (CL07171) \\ 3: Transcription factor Bmr1 (CL07172) \\ 4: Hydroxynaphthalene reductase (CLO7173) \\ 5: Ribosome biogenesis protein (CL07174) \\ 6: ADP-ribosylation factor-binding protein (CL07175) \\ 7: Hypothetical protein (CL07176) \\ 8: Phosphatidylinositol glycan (CL07177) \\ 9: Exosome complex exonuclease (CL07178)}

10: Hypothetical protein (CL07179)

11: Nucleolar protein (CL07180)

12: Prenylcysteine oxidase (CL07181)

13: Cytochrome P450 (CL07182)

14: Hypothetical protein (CL07183)

15: Translation initiation factor IF-2 (CL07184)

16: Hypothetical protein (CL07185)

17: ABC transporter (CL07186)

Fig. 11. CLT1 regulates melanin and toxin formation. A, RNA-seq analysis reveals a significantly high transcript abundance of genes involved in melanin biosynthesis. The diagram shows the process of fungal melanin biosynthesis as described by Soanes et al. (2012). For each enzyme, the histograms show the abundance of transcripts encoding enzymes in melanin biosynthesis, gene expression levels calculated by log 2 reads per kilobase million of transcript abundances in T806 compared with CX-3. B, The 57.89-kb toxin synthesis cluster of genes in the Curvularia lunata CX-3 genome. Genes downregulated in T806 compared with CX-3 are highlighted in green. Genes upregulated in T806 compared with CX-3 are highlighted in red. A gene with no expression change is highlighted in white. 
$57.89 \mathrm{~kb}$ on scaffold 9 of the $C$. lunata $\mathrm{CX}-3$ genome, which is similar to the cercosporin-associated gene cluster of Cercospora nicotianae (Soanes et al. 2012).

The process of toxin synthesis and causing disease in pathogenic fungi involves active translocation of molecules from hyphae to the cell environment, and the observed decreased expression in this study of $\mathrm{ABC}$ transport protein might reflect such a scenario. The mechanism of these predicted toxin biosynthesis genes should be researched further. In addition, the conidiation of $\Delta$ clxyn $24, \Delta$ claxe 43 , and $\Delta$ clxyn $24 \&$ claxe 43 was reduced as well as that of $\Delta$ clt1 (Table 1).

Acetyl-CoA carrying acetate is used to build up large biological molecules, such as precursors to sterols, polyketides, and long-chain fatty acids (Asakura et al. 2012). As the melanin and toxin biosynthetic pathway begins from malonyl-CoA derived from acetyl-CoA, the acetyl-CoA derived from xylan degradation is considered essential for melanin and toxin production in C. lunata (Ni et al. 2017). The polyketide pathway is an important metabolic process in filamentous fungi, which is involved in growth, development, and pathogenicity. Reports suggested that $P K S 1$ and $P K S 2$ were responsible for the biosynthesis of T-toxin in Bipolaris maydis (Baker et al. 2006). In C. lunata, PKS18 was functionally characterized to encode a PKS that converts malonyl-CoA to $1,3,6,8$-THN in the DHN melanin synthesis pathway. The deletion of pks 18 also results in the defect of toxin production and hyphal melanization in C. lunata (Gao and Chen 2017), which was consistent with the phenotype of clxyn 24 and claxe 43 mutants. However, the conidiation of $\Delta p k s 18$ was not different from that of the WT, which means
PKS18 was not responsible for conidiation in C. lunata (Gao and Chen 2017).

These findings suggest that an unknown regulatory pathway may connect melanin and toxin synthesis to acetyl-CoA generated by glycolysis in C. lunata, and melanin and toxin may have a coordinate expression during the infection process (Gao et al. 2015a). Above all, this study indicated that Clt1 physically interacted with Clxyn 24 and Claxe43 via its BTB domain, which acts upstream of xylan metabolism. The utilization of xylan provides acetyl-CoA for the synthesis of melanin and toxin, energy, and other intermediate metabolites for conidiation (Fig. 12).

\section{MATERIALS AND METHODS}

Fungal strains, plant materials, and growth conditions.

C. lunata WT strain CX-3 was used as a recipient for the transformation experiment. Unless mentioned otherwise, all strains were grown on solid xylan medium at $28^{\circ} \mathrm{C}$. Nicotiana benthamiana and Zea mays were grown under $16 \mathrm{~h}$ of light and $8 \mathrm{~h}$ of dark at $24^{\circ} \mathrm{C}$.

\section{Y2H screening and sequence analysis.}

$\mathrm{Y} 2 \mathrm{H}$ screening and sequence analysis were performed as described (Gao et al. 2015d). The sequences of primers are listed in Supplementary Table S3. Phylogenetic trees were constructed using MEGA 5.0 and alignment was conducted using ClustalW.

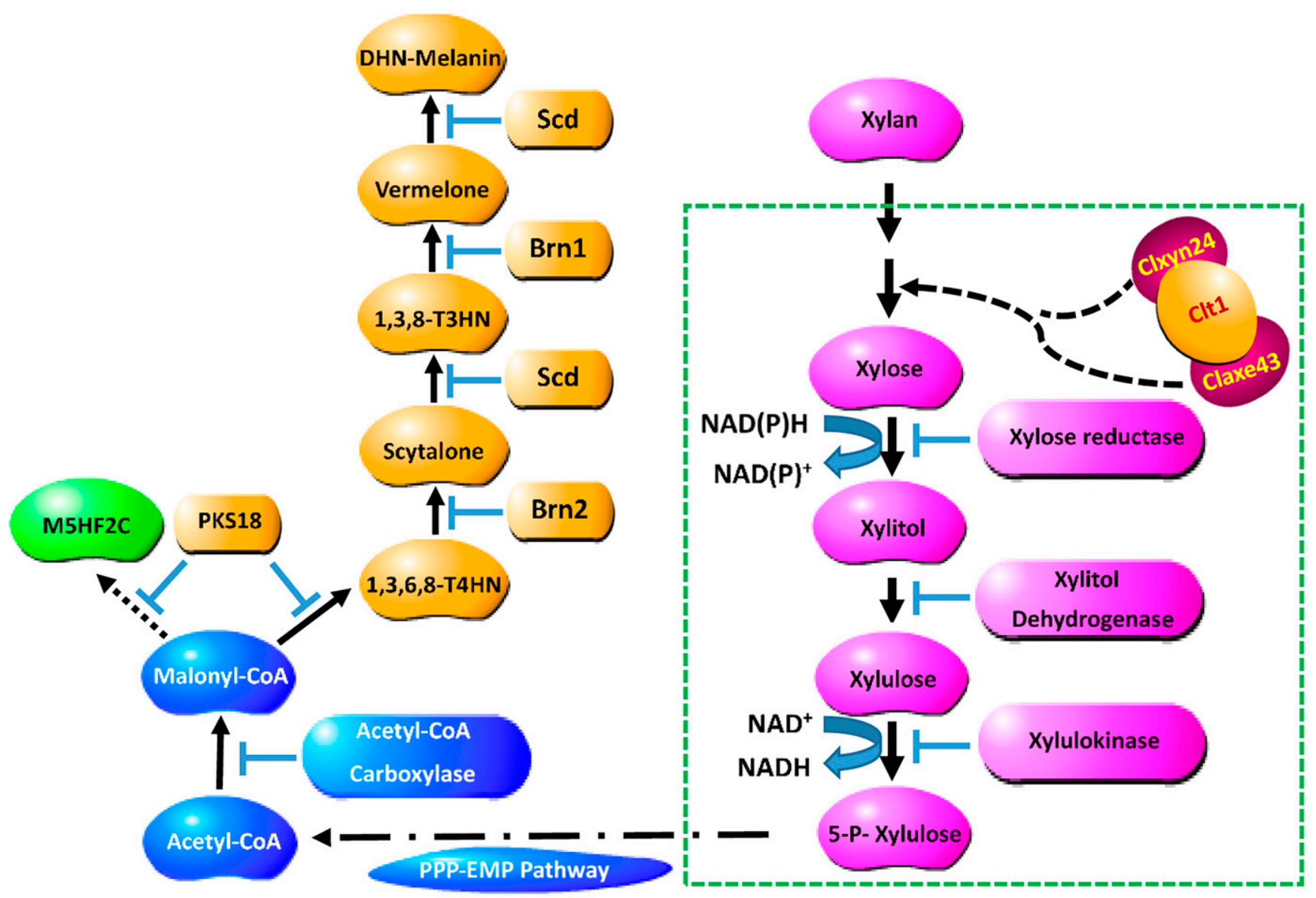

Fig. 12. Xylan metabolism provides acetyl-CoA for melanin and toxin biosynthesis in Curvularia lunata. Clt1 physically interacted with Clxyn24 and Claxe43 to degrade xylan, which was used as a carbon source of $C$. lunata growth. The utilization of xylan provides acetyl-CoA for the synthesis of melanin and toxin. The magenta graphics represent the xylan degradation pathway, whereas the orange graphics represent the melanin synthesis pathway. 
Target Y2H assays.

The full-length cDNA sequences of CLT1, CLXYN24, and CLAXE43 were inserted into the EcoRI-BamHI sites of the pGADT7 and pGBKT7 vectors (Clontech), respectively. Target $\mathrm{Y} 2 \mathrm{H}$ assays were conducted as described (Gao et al. 2017).

\section{BiFC assays.}

The cDNA sequences of CLT1, CLXYN24, and CLAXE43 were cloned via the $\mathrm{pDONR} / Z$ eo vector into the corresponding pEarleyGate202-YN or pEarleyGate201-YC vector using the Gateway cloning system (Life Technologies). BiFC assays were conducted as described (Gao et al. 2017).

\section{CLXYN24 and CLAXE43 deletion.}

The CLXYN24 and CLAXE43 genes were deleted out of the CX-3 genome. clxyn24\&claxe 43 deletion mutant was accomplished by deletion of CLAXE43 out of the genome of $\Delta$ clxyn 24 (Supplementary Fig. S4). Target genes were deleted using Agrobacterium tumefaciens-mediated transformation method. PCR and Southern hybridization pattern were employed to confirm the homologous recombination at CLXYN24 or CLAXE43 locus (Gao et al. 2014a; Yu et al. 2014).

\section{Analysis of growth rate, conidiation, and virulence.}

The analysis of growth rate, conidiation, and virulence were conducted as described (Gao et al. 2017; Wang et al. 2017).

Prokaryotic expression and analysis of xylan degradation.

The prokaryotic expression was analyzed by digesting the open reading frames of Clt1, ClXyn24, and ClAxe43, respectively, and ligating with plasmid pET28a. After verification of PCR and DNA sequencing, the Clt1, ClXyn24, and ClAxe43 proteins fused with $6 \mathrm{x}$ His tag in pET28a plasmid were respectively transferred into Escherichia coli BL21 (DE3) to which 1 $\mathrm{mM}$ isopropyl- $\beta$-D-thiogalactoside was added to induce gene expression. The soluble fusion proteins were analyzed by Western blot and were purified through Ni-chelating affinity chromatography. Xylan was treated with different combinations of proteins, the release of xylose was measured using the dinitrosalicylic acid method (Saravanakumar et al. 2016).

\section{Pigmentation.}

The pigmentation of hyphae on xylan or xylose solid medium were tested according to the description of the procedure (Gao et al. 2017). The WT strain (CX-3), clt1 deletion mutant ( $\Delta$ clt1), clxyn24 deletion mutant $(\Delta$ clyn 24$)$, claxe43 deletion mutant (Aclaxe43), and clxyn24 claxe43 double deletion mutant ( $\Delta$ clyn $24 \&$ claxe 43$)$ were cultured on the xylan or xylose palates for 21 days to observe the pigmentation. The samples cultured in xylan or xylose liquid medium for 9 days were collected for qRT-PCR analyses of CMR1, PKS18, BRN1, $B R N 2$, and $S C D$. The genes were expressed as fold change compared with that of WT.

\section{Analysis of M5HF2C toxin production.}

The mutants were cultured in Fries 3 medium with xylan or xylose as a carbon source for 30 days, to determine whether they retained the ability to produce the M5HF2C toxin. Highpressure liquid chromatography (HPLC) and mass spectrum analysis of the extract from $C$. lunata cultures was performed on an Agilent 1100 HPLC station, to determine the amount of M5HF2C, according to the previously described procedure (Gao et al. 2017; Liu et al. 2009).

\section{RNA-seq library preparation and construction.}

C. lunata $\mathrm{CX}-3$ and the toxin production-deficient mutant T806 were used for RNA-seq analysis. Transcriptomes of CX-3 and T806 served as the control and test group, respectively. The total RNA was prepared as previously described (Gao et al. $2014 b$ ). The libraries were constructed by 1 Gene. The genes with an FDR cutoff $\leq 0.001$ and $P \leq 0.05$ were considered as significantly differentially expressed genes.

\section{Analysis of gene expression and cluster.}

Gene expression, cluster, principal component analysis, and CAST analyses were performed by 1 Gene, using the MultiExperiment Viewer with threshold affinity value 0.9 and other default parameters (Saeed et al. 2003).

\section{ACKNOWLEDGMENTS}

We thank C. Xue, College of Plant Protection, Shenyang Agricultural University for his encouragement during the revision of this article and 1 Gene for providing technical assistance with bioinformatics.

\section{AUTHOR-RECOMMENDED INTERNET RESOURCE}

Weblogo: http://weblogo.berkeley.edu

\section{LITERATURE CITED}

Asakura, M., Yoshino, K., Hill, A. M., Kubo, Y., Sakai, Y., and Takano, Y. 2012. Primary and secondary metabolism regulates lipolysis in appressoria of Colletotrichum orbiculare. Fungal Genet. Biol. 49:967975.

Baker, S. E., Kroken, S., Inderbitzin, P., Asvarak, T., Li, B. Y., Shi, L., Yoder, O. C., and Turgeon, B. G. 2006. Two polyketide synthase-encoding genes are required for biosynthesis of the polyketide virulence factor, T-toxin, by Cochliobolus heterostrophus. Mol. Plant-Microbe Interact. 19:139-149.

Chumley, F. G., and Valent, B. 1990. Genetic analysis of melanin-deficient, nonpathogenic mutants of Magnaporthe grisea. Mol. PlantMicrobe Interact. 3:135-143.

Condon, B. J., Leng, Y., Wu, D., Bushley, K. E., Ohm, R. A., Otillar, R., Martin, J., Schackwitz, W., Grimwood, J., MohdZainudin, N., Xue, C., Wang, R., Manning, V. A., Dhillon, B., Tu, Z. J., Steffenson, B. J., Salamov, A., Sun, H., Lowry, S., LaButti, K., Han, J., Copeland, A., Lindquist, E., Barry, K., Schmutz, J., Baker, S. E., Ciuffetti, L. M., Grigoriev, I. V., Zhong, S., and Turgeon, B. G. 2013. Comparative genome structure, secondary metabolite, and effector coding capacity across Cochliobolus pathogens. PLoS Genet. 9:e1003233.

Degefu, Y., Paulin, L., and Lubeck, P. 1997. Cloning, sequencing and expression of a xylanase gene from the maize pathogen Helminthosporium turcicum. Eur. J. Plant Pathol. 107:457-465.

Eliahu, N., Igbaria, A., Rose, M. S., Horwitz, B. A., and Lev, S. 2007. Melanin biosynthesis in the maize pathogen Cochliobolus heterostrophus depends on two mitogen-activated protein kinases, Chk1 and Mps1, and the transcription factor Cmr1. Eukaryot. Cell 6:421-429.

Fox, E. M., Gardiner, D. M., Keller, N. P., and Howlett, B. J. 2008. A $\mathrm{Zn}(\mathrm{II})_{2} \mathrm{Cys}_{6}$ DNA binding protein regulates the sirodesmin PL biosynthetic gene cluster in Leptosphaeria maculans. Fungal Genet. Biol. 45: 671-682.

Gao, J. X., and Chen, J. 2017. Involvement of a polyketide synthetase CIPKS18 in the regulation of vegetative growth, melanin and toxin synthesis, and virulence in Curvularia lunata. Plant Pathol. J. 33:597-601.

Gao, J. X., Jing, J., and Chen, J. 2015a. Elementary coordinated expression research on genes related to the synthesis of pathogenesis-related melanin and toxin in Cochliobolus lunatus. J. SJTU.Agro Sci. 33:53-58.

Gao, J. X., Jing, J., Liu, T., and Chen, J. 2015b. Identification of Clt-1-regulated proteins associated with the production of non-host-specific toxin and pathogenicity in Cochliobolus lunatus. J. Phytopathol. 163:33-41.

Gao, J. X., Jing, J., Liu, T., Yu, C. J., Li, Y. Q., and Chen, J. 2015 c. Identification of proteins associated with the production of melanin and with pathogenicity in maize pathogen Curvularia lunata. Australas. Plant Pathol. 44:599-603.

Gao, J. X., Jing, J., Yu, C. J., and Chen, J. 2015d. Construction of a high-quality yeast two-hybrid library and its application in identification of interacting proteins with Brn1 in Curvularia lunata. Plant Pathol. J. 31:108-114.

Gao, J. X., Liu, T., and Chen, J. 2014a. Insertional mutagenesis and cloning of the gene required for the biosynthesis of the non-host-specific 
toxin in Cochliobolus lunatus that causes maize leaf spot. Phytopathology 104:332-339.

Gao, J. X., Yu, C. J., Wang, M., Sun, J. N., Li, Y. Q., and Chen, J. 2017. Involvement of a velvet protein $\mathrm{ClVelB}$ in the regulation of vegetative differentiation, oxidative stress response, secondary metabolism, and virulence in Curvularia lunata. Sci. Rep. 7:46054.

Gao, S., Li, Y., Gao, J., Suo, Y., Fu, K., Li, Y., and Chen, J. 2014b. Genome sequence and virulence variation-related transcriptome profiles of Curvularia lunata, an important maize pathogenic fungus. BMC Genomics 15:627.

Gao, S., Liu, T., Li, Y., Wu, Q., Fu, K., and Chen, J. 2012. Understanding resistant germplasm-induced virulence variation through analysis of proteomics and suppression subtractive hybridization in a maize pathogen Curvularia lunata. Proteomics 12:3524-3535.

Inderbitzin, P., Asvarak, T., and Turgeon, B. G. 2010. Six new genes required for production of T-toxin, a polyketide determinant of high virulence of Cochliobolus heterostrophus to maize. Mol. PlantMicrobe Interact. 23:458-472.

Keller, N. P., and Hohn, T. M. 1997. Metabolic pathway gene clusters in filamentous fungi. Fungal Genet. Biol. 21:17-29.

Khan, I. D., Makkar, A., Malik, A., Khan, S., Mehdi, I., Arif, S., Aden, D., Somayaji, P. and Roomi, K. 2017. Curvularia keratomycosis after cataract surgery. J. Arch. Mil. Med. 5:e57331.

Rižner, T., and Wheeler, M. H. 2003. Melanin biosynthesis in the fungus Curvularia lunata (teleomorph: Cochliobolus lunatus). Can. J. Microbiol. 49:110-119.

Liu, T., Liu, L. X., Jiang, X., Huang, X. L., and Chen, J. 2009. A new furanoid toxin produced by Curvularia lunata, the causal agent of maize Curvularia leaf spot. Can. J. Plant Pathol. 31:22-27.

Liu, T., Wang, Y., Ma, B., Hou, J., Jin, Y., Zhang, Y., Ke, X., Tai, L., Zuo, Y., and Dey, K. 2016. Clg2p interacts with Clf and ClUrase to regulate appressorium formation, pathogenicity and conidial morphology in Curvularia lunata. Sci. Rep. 6:24047.

Liu, T., Xu, S. F., Liu, L. X., Zhou, F. H., Hou, J. M., and Chen, J. 2011. Cloning and characteristics of Brnl gene in Curvularia lunata causing leaf spot in maize. Eur. J. Plant Pathol. 131:211-219.

Ni, X., Gao, J. X., Yu, C. J., Wang, M., Sun, J. N., Li, Y. Q., and Chen, J. 2017. MAPKs and acetyl-CoA are associated with Curvularia lunata pathogenicity and toxin production in maize. J. Integr. Agric. 16: 60345-60347.
Perez-Torrado, R., Yamada, D., and Defossez, P. A. 2006. Born to bind: The BTB protein-protein interaction domain. BioEssays 28:1194-1202.

Saeed, A. I., Sharov, V., White, J., Li, J., Liang, W., Bhagabati, N., Braisted, J., Klapa, M., Currier, T., Thiagarajan, M., Sturn, A., Snuffin, M., Rezantsev, A., Popov, D., Ryltsov, A., Kostukovich, E., Borisovsky, I., Liu, Z., Vinsavich, A., Trush, V., and Quackenbush, J. 2003. TM4: A free, open-source system for microarray data management and analysis. Biotechniques 34:374-378.

Saravanakumar, K., Fan, L., Fu, K., Yu, C., Wang, M., Xia, H., Sun, J., Li, Y., and Chen, J. 2016. Cellulase from Trichoderma harzianum interacts with roots and triggers induced systemic resistance to foliar disease in maize. Sci. Rep. 6:35543.

Soanes, D. M., Chakrabarti, A., Paszkiewicz, K. H., Dawe, A. L., and Talbot, N. J. 2012. Genome-wide transcriptional profiling of appressorium development by the rice blast fungus Magnaporthe oryzae. PLoS Pathog. 8:e1002514.

Wang, F., Gao, W., Sun, J., Mao, X., Liu, K., Xu, J., Fu, D., Yuan, M., Wang, H., Chen, N., Xiao, S., and Xue, C. 2020. NADPH oxidase CINOX2 regulates melanin-mediated development and virulence in Curvularia lunata. Mol. Plant-Microbe Interact. 33:13151329

Wang, M., Wang, S. Q., Ma, J., Yu, C. J., Gao, J. X., and Chen, J. 2017. Detection of Cochliobolus heterostrophus races in South China. J. Phytopathol. 165:681-691.

Wang, X. H., Gao, J. X., Gao, S. G., Liu, T., Lu, Z. X., Li, Y. Q., and Chen, J. 2019. Research progress of Curvularia leaf spot in maize. Acta Phytopathol. Sin. 49:433-444.

Xu, S. F., Chen, J., Liu, L. X., Wang, X. F., Huang, Y. L., and Zhai, Y. H. 2007. Proteomics associated with virulence dierentiation of Curvularia lunata in maize (Zea maydis) in China. J. Integr. Plant Biol. 49:487-496.

Yu, C., Fan, L., Wu, Q., Fu, K., Gao, S., Wang, M., Gao, J., Li, Y., and Chen, J. 2014. Biological role of Trichoderma harzianum-derived platelet-activating factor acetylhydrolase (PAF-AH) on stress response and antagonism. PLoS One 9:e100367.

Zollman, S., Godt, D., Privé, G. G., Couderc, J. L., and Laski, F. A. 1994. The BTB domain, found primarily in zinc finger proteins, defines an evolutionarily conserved family that includes several developmentally regulated genes in Drosophila. Proc. Natl. Acad. Sci. U.S.A. 91:10717-10721. 